Revistade
Economida
Contemporâned

\title{
O IMPACTO DOS INVESTIMENTOS EM P\&D NO DESEMPENHO DAS EMPRESAS: APLICAÇÕES NO USO DE REGRESSÃO QUANTÍLICA COM VARIÁVEIS INSTRUMENTAIS
}

\author{
Leonardo Andrade Rocha ${ }^{a}$ \\ Ahmad Saeed Khan ${ }^{b}$ \\ Patrícia Verônica Pinheiro Sales Lima ${ }^{c}$ \\ Maria Ester Soares Dal Poz $z^{d}$ \\ Carlos Alano Soares de Almeida

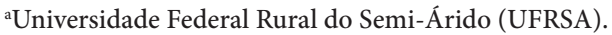 \\ 'Universidade Federal do Ceará (UFC). \\ 'Universidade Federal do Ceará (UFC). \\ ${ }^{d}$ Faculdade de Ciências Aplicadas da Universidade Estadual de Campinas (FAC/UNICAMP). \\ ${ }^{e}$ Universidade Federal Rural do Semi-Árido (UFRSA).
}

Artigo recebido em 21/07/2017 e aceito para publicação em 03/09/2018.

RESUMO: O estudo analisa o impacto dos investimentos em P\&D nas vendas das firmas, tomando uma amostra de 2.000 firmas. Usando a técnica de variáveis instrumentais na regressão quantílica, o coeficiente de elasticidade em $P \& D$ apresentou-se consideravelmente maior em relação ao método tradicional (estimador de Koenker e Bassett) e com inversão de tendência com aumento dosquantis. O estimador de regressão quantílica com variáveis instrumentais revela os riscos de tendenciosidade e inconsistência quando o pressuposto de exogeneidade é violado. Ao contrário dos resultados apresentados na literatura, as implicações deste estudo mostram que firmas com maior volume nas vendas (quantis superiores da distribuição condicional) obtêm maior retorno no uso dos investimentos em P\&D.

PALAVRAS-CHAVE: inovação; crescimento; P\&D; regressão quantílica.

CLASSIFICAÇÃO JEL: C21; O32; O40; Q55.

Correspondência para: Patrícia Verônica Pinheiro Sales Lima

Contato: patricialima@ufc.br 


\section{THE IMPACT OF INVESTMENTS IN R\&D ON FIRM PERFORMANCE: APPLICATIONS OF QUANTILE REGRESSION WITH INSTRUMENTAL VARIABLES}

ABSTRACT: This study analyzes the impact of R\&D in firms' sales levels, taking a sample of 2,000 firms. Using the technique of instrumental variables in the quantile regression, the coefficient of elasticity in R\&D demonstrated to be considerably higher compared to the traditional method (Koenker and Bassett estimator) and a trend reversal with rising quantile. The estimator of the quantile regression with instrumental variables reveals the risks of bias and inconsistency when the exogeneity assumption is violated. Unlike the results presented in the recent literature, the implications of this study show that firms with higher sales (higher quantis of conditional distribution) obtain higher returns on the use of R\&D investments.

KEYWORDS: innovation; growth; R\&D; quantile regression. 


\section{INTRODUÇÃO}

O estudo da relação entre os esforços em inovação e o desempenho da empresa oferece um importante campo de atuação da política econômica: primeiramente, no subsídio de importantes recursos destinados a reforçar uma parcela significativa das atividades de pesquisa; em segundo, na própria manutenção dos níveis de emprego, via aumento na demanda por parte das firmas inovadoras e; em terceiro, na construção de trajetórias tecnológicas, garantindo condições de crescimento em longo prazo por meio do progresso técnico (HALL, LOTTI e MAIRESSE, 2013).

Esta compreensão torna-se importante para destacar a ampla contribuição destes investimentos no desenvolvimento de muitos países. Os tomadores de decisão da política econômica encontram-se especialmente interessados nos retornos sociais advindos destes investimentos. Neste sentido, muitos estudos de crescimento vêm enfatizando o papel do progresso técnico nas condições de crescimento em longo prazo (AGHION e HOWITT, 2009).

Por outro lado, os gestores das empresas frequentemente centram suas avaliações nos retornos privados, sendo estes condicionados aos incentivos cujas empresas enfrentam para a realização de tais investimentos. Em cada caso, o resultado obtido a partir da aplicação destes recursos depende de importantes fatores tais como: economias de escala, escopo, desempenho no setor em que atuam, situar-se na fronteira ou afastado desta etc (COAD, 2008, 2011).

Desta forma, as técnicas tradicionais de regressão linear deixam de capturar estes dentre outros importantes fatores: a heterogeneidade dos efeitos destes investimentos entre os diferentes tipos de firmas. Baseado nestas restrições, um número crescente de estudos vem incorporando em suas análises o impacto da heterogeneidade entre as firmas na relação entre P\&D e desempenho (COAD e RAO, 2011; HÖLZL, 2009; FALK, 2012; MENDES, SERRASQUEIRO e NUNES, 2014; MONTRESOR e VEZZANI, 2015).

Uma importante técnica paramétrica consiste na abordagem de regressões quantílicas, de acordo com as contribuições de Koenker e Bassett (1978). Esta técnica, robusta quanto à presença de dados discrepantes (outliers) e de erros não distribuídos normalmente, permite estimar relações entre as variáveis diferentes das medidas de tendência central. Desta forma, o método investiga partes específicas da distribuição condicional da variável dependente, capturando diferenças nos efeitos dos investimentos conforme diferentes quantis da distribuição.

Conforme Coad (2008, 2011), a atividade inovativa das empresas incorpora importantes diferenças cujas regressões quantílicas permitem analisá-las com maior precisão. Isto porque os investimentos em $\mathrm{P} \& \mathrm{D}$ geram resultados diferentes entre as fir- 
mas que, em muitas circunstâncias, estão relacionadas com a posição da firma em relação à fronteira do setor. O sucesso da inovação desloca a fronteira tecnológica, melhorando a vantagem competitiva das firmas que se situam na sua vizinhança, por meio do conhecimento privilegiado acerca dos novos produtos e das técnicas produtivas. Assim, partes da distribuição condicional retratam aspectos tecnológicos bastante distintos, sejam nas firmas da fronteira ou mais afastadas, de acordo com o resultado obtido a partir da aplicação do investimento (COAD e RAO, 2011).

Recentemente, Montresor e Vezzani (2015) retrataram uma importante contribuição ao tema, apresentando evidências empíricas de como as inovações podem apresentar resultados distintos na função de produção das empresas. Como pilar básico na moderna teoria da economia da inovação, firmas de diferentes tamanhos apresentam capacidades intrinsecamente diversas de introdução, apropriação e exploração das suas inovações, de maneira que a heterogeneidade entre as firmas corresponde a um resultado da própria estrutura industrial e da sua dinâmica (COHEN, 2010). Empregando a técnica de regressão quantílica em uma amostra de firmas que representam os top investidores em P\&D no mundo, foi estimada a função da produção relacionando as receitas das firmas como função dos estoques de capital, mão de obra e do estoque de conhecimento (representado pelo recurso em P\&D aplicado). Os resultados estimados apontaram para um padrão decrescente no coeficiente de elasticidade da pesquisa nas receitas ao longo dos quantis condicionais. Firmas situadas nos quantis inferiores apresentam se beneficiar mais com o investimento em P\&D aplicado em relação às firmas situadas nos quantis superiores. Mesmo distinguindo a amostra por setores de alta tecnologia, média tecnologia e baixa tecnologia, o padrão decrescente no coeficiente de elasticidade da pesquisa ainda persiste. A heterogeneidade do resultado da pesquisa reflete possíveis custos de oportunidades entre os recursos, sinalizando para um padrão de substitutibilidade entre tangível e intangível, que é capturado ao longo da evolução dos quantis condicionais.

Além disso, as estimativas dos parâmetros a partir da técnica de regressão quantílica revelam elementos importantes de heterogeneidade que são capturados em diferentes pontos da distribuição condicional, cujas técnicas tradicionais ocultam ou mesmo deixam de trabalhá-las de forma mais adequada no modelo. Nesse caso, a técnica torna-se fundamental nos modelos de economia da inovação, cujas assimetrias na distribuição ajudam a explicar possíveis diferenças no retorno dos investimentos (COAD, SEGARRA e TERUEL, 2016).

Contudo, os ajustes nos investimentos em P\&D são afetados pelos níveis de lucros e pelo próprio crescimento, seguindo um importante campo da teoria do investimento proposto por Eisner (1978) e recentemente tratada nos modelos de crescimento endógenos em Aghion e Howitt (2009), Aghion et al. (2012), Aghion, Hémous e Kharroubi 
(2014), Aghion e Jaravel (2015), Aghion, Howitt e Prantl (2015) e Aghion e Festré (2017). A premissa em tais estudos consiste no fato dos investimentos em pesquisa estarem sujeitos a incertezas de longo prazo, tornando a tomada de decisão susceptível às condições de apropriabilidade. Tanto o aprendizado quanto a inovação tornam-se endógenas na indústria, de maneira que tais habilidades são baseadas nas competências incorporadas nas firmas, nos conhecimentos interno e externo que podem ser aplicados e nas suas rotinas construídas (DOSI, 1982; DOSI, MARENGO e PASQUALI, 2006; DOSI, GAFFARD e NESTA, 2008; TEECE, 2009). Por fim, a estrutura que "molda" a indústria assim como tais "moldes" retornam para as firmas, conduzindo a diferenças nos retornos dos investimentos que sustentam as assimetrias entre as firmas (PIRES, 2009).

Entretanto, tais influências são desconsideradas nos modelos de regressão quantílica que buscam capturar uma melhor forma de medir a heterogeneidade presente entre as firmas. Recentes avanços na abordagem de funções quantílicas, destacando Chernozhukov e Hansen $(2006 ; 2008)$, propulseram um estimador alternativo que visa controlar a endogeneidade por meio da técnica de variáveis instrumentais. Na presença de regressores endógenos, o estimador de Koenker a Bassett (1978) é tendencioso e inconsistente. Assim, as estimativas de regressão quantílica tornam-se imprecisas para avaliar a verdadeira magnitude do ganho econômico a partir das aplicações dos investimentos em P\&D.

Como Bogliacino (2014), Montresor e Vezzani (2015), Kancs e Siliverstovs (2016) e Castellani et al. (2017), o presente estudo empregou uma amostra dos principais investidores em P\&D para analisar a relação entre os investimentos em P\&D e o desempenho das firmas, controlando efeitos de endogeneidade que visem os resultados apresentados pela literatura. Na ausência do controle de endogeneidade, os coeficientes de elasticidade da pesquisa nas receitas convergem para os resultados apresentados em Montresor e Vezzani (2015). Após o devido controle, o padrão crescente com os quantis condicionais sugere que as firmas com melhor desempenho (quantis superiores da distribuição condicional) obtêm maiores resultados a partir do uso dos investimentos, indicando um retorno maior em relação às firmas situadas nos quantis inferiores.Tais evidências corroboram com a hipótese de Cohen e Levinthal (1989) sobre capacidade absortiva, segundo a qual o aumento das habilidades das firmas em assimilar e transformar o conhecimento advindo do ambiente em que atuam acarreta no aumento da sua capacidade absortiva. Nesse caso, firmas situadas nos quantis superiores da distribuição obtêm maiores retornos que podem estar associados a um padrão distinto de cumulatividade do conhecimento em relação às firmas situadas na parte inferior da distribuição condicional. Essa relação tem sido recentemente tratada no conceito de "aproximação com a fronteira tecnológica" a partir das contribuições de Griffith, Red- 
ding e Van Reenen (2003; 2004) e Aghion e Jaravel (2015), em que os spillovers da pesquisa tendem a ser mais apropriados na medida em que as firmas se aproximam da fronteira tecnológica. Esse aumento nas condições de apropriabilidade tornam a aplicação dos investimentos em pesquisa mais diferenciados entre as firmas.

A presente pesquisa contribui ao debate sobre o tema, destacando a contribuição em cinco pontos:

1. Ao contrário dos resultados apresentados em Montresor e Vezzani (2015), a aplicação dos investimentos em pesquisa gera retornos maiores nas firmas com maior desempenho (situadas nos quantis superiores da distribuição condicional), uma vez que controlamos importantes fatores associados ao desempenho e que condicionam a demanda por investimentos;

2. O padrão crescente nos coeficientes de elasticidade $\mathrm{P} \& \mathrm{D}$-vendas ao longo dos quantis sugere uma confluência com os resultados apresentados por Griffith, Redding e Van Reenen (2003; 2004) e Aghion e Jaravel (2015) sobre "proximidade com a fronteira" e externalidades da pesquisa;

3. Firmas situadas nos quantis superiores tendem a apresentar um padrão mais evidente de apropriação da pesquisa, aumentando o retorno sobre o recurso aplicado;

4. Este padrão crescente pode estar associado a um possível efeito Cohen-Levinthal de capacidade absortiva, cujas firmas com maior desempenho administram condições de maior apropriabilidade com seus esforços em inovação, conforme Breschi, Malerba e Orsenigo (2000);

5. Tais resultados apontam para um importante papel sobre o debate das fontes de convergência e divergência entre os países e sobre a formação de políticas apropriadas que maximizam o crescimento local. Neste caso, spillovers da pesquisa apresentam padrões heterogêneos entre firmas com maior e menor desempenho, assim como países avançados e menos avançados, tornando seu efeito uma "força subjacente" ao processo de convergência entre as nações.

\section{METODOLOGIA EMPÍRICA}

\subsection{DEFINIÇÃO DA AMOSTRA}

Para medir a influência dos esforços inovativos no desempenho das firmas, adotou-se a base de dados do The 2013 EU Industrial R\&D Investment Scoreboard (COMMISSION, 2013). Esse relatório é publicado, anualmente, desde 2004, fornecendo o ranking das empresas com o maior volume de gastos em Pesquisa \& Desenvolvimento (P\&D), distri- 
buídas em 40 setores e 46 países. As informações incluem, além desta variável, o volume das vendas (em € milhões), número de empregados, despesas com capital (em $€$ milhões), rentabilidade, além das medidas de crescimento. Os dados do relatório são apresentados conforme uma defasagem de um ano. Dessa forma, os dados apresentados no relatório de 2013 representam os valores do ano anterior.

$\mathrm{O}$ relatório concentra suas análises nos dois mil maiores investidores em $\mathrm{P} \& \mathrm{D}$ aplicado, cujos recursos somados representam uma quantia investida superior a $€ 22,6$ bilhões em recurso de pesquisa, somente no ano 2012. A amostra abrange empresas com sede na União Europeia (527 firmas), EUA (658 firmas), Japão (353 firmas) e outros países (462 firmas), incluindo China, Taiwan, Coréia do Sul, Suíça, Ilhas Cayman, Índia, Canadá, Austrália, Israel, Noruega, Bermudas, Brasil e outros 13 países.

Esta base vem sendo recentemente empregada em estudos empíricos no contexto de inovação em decorrência da representatividade dos dados acerca do tema (BOGLIACINO, 2014; MONTRESOR e VEZZANI, 2015; KANCS e SILIVERSTOVS, 2016; CASTELLANI et al., 2017). Isto deriva da base de dados contemplar importantes informações sobre geografia e setorial (ver Anexo, Tabelas 9 e 10), de maneira que as firmas selecionadas representam os top investidores em inovação e concentrando aproximadamente $90 \%$ de todo o investimento em P\&D no mundo aplicado (COMMISSION, 2013, p.11).

\subsection{REGRESSÃO QUANTÍLICA COM VARIÁVEIS INSTRUMENTAIS}

Uma das maiores vantagens dos modelos de regressão quantílica consiste em caracterizar o impacto heterogêneo entre as variáveis conforme diferentes pontos da distribuição condicional. Esta habilidade de tratamento pelo estimador vem ganhando muito destaque em diversas aplicações econômicas ${ }^{1}$.

O estimador de regressão quantílica proposto por Koenker e Bassett (1978) parte do pressuposto que os regressores do modelo são exogenamente determinados ao longo da distribuição condicional da variável dependente. Desta maneira, em cada quantil condicional, as informações capturadas pelos regressores são completas, de forma que o pressuposto de ortogonalidade entre os regressores e a perturbação estocástica não é violado.

Ao contrário disto, em situações particulares, o pressuposto de ortogonalidade não é sustentável, de maneira que o estimador tradicional de regressão quantílica tor-

1 Para aplicações de regressão quantílica em modelos financeiros, ver Coad (2011) e Coad e Rao (2011). 
na-se inconsistente e, portanto, inapropriado para capturar o real efeito entre as variáveis (regressores e regressando) ao longo dos diferentes pontos da distribuição condicional. Para solucionar este problema, é imposto ao modelo condições que limitam a evolução dos dados ao longo dos quantis condicionais. A partir disto, sem hipóteses de forma funcional conhecida, estas condições limitantes são obtidas por meio da técnica de variáveis instrumentais, buscando estimar os verdadeiros parâmetros em cada quantil, superando, assim, o problema de endogeneidade entre os regressores.

O método aplicado neste estudo segue as contribuições recentemente propostas por Chernozhukov e Hansen $(2004,2008)$. Na abordagem tradicional de regressão linear, um modelo de regressão quantílica para um dado quantil condicional pode ser definido da seguinte forma:

$$
\begin{aligned}
& Q_{\tau}(Y \mid X)=X^{\prime} \beta(\tau) \therefore \tau \in(0,1) \\
& Y=Q_{\tau}(Y \mid X)+\varepsilon(\tau)
\end{aligned}
$$

Conforme a equação (1), $Q_{\tau}(\mid)$ denota a função quantil condicional par o $\tau$-é-simo quantil da distribuição. Nesta abordagem tradicional, o valor esperado $E(X \mid \varepsilon(\tau), \tau)=0$, de forma que o estimador proposto por Koenker e Bassett (1978) é consistente e não tendencioso. Entretanto, em determinadas situações, este pressuposto é violado, o que torna o estimador de $\beta(\tau)$ inapropriado em casos de variáveis endógenas.

Pensando nisso, Chernozhukov e Hansen (2004, 2006, 2008) propuseram um estimador alternativo em casos de violação do pressuposto:

$$
\begin{gathered}
S_{U}(Y \mid D, X)=D^{\prime} \alpha(U)+X^{\prime} \beta(U) \\
D=\delta(X, Z, V(U)) \\
U \mid X, Z \sim \text { Uniforme }(0,1) \\
\tau \mapsto D^{\prime} \alpha(\tau)+X^{\prime} \beta(\tau)
\end{gathered}
$$

Conforme as equações (2a) a $(2 \mathrm{~d}), S_{U}(Y \mid D, X)$ representa a função quantil condicional, com destaque para a inclusão do vetor de variável endógena $(D)$. A variável endógena, por meio de uma forma funcional não conhecida, é afetada pelos regressores exógenos $X$ e por um conjunto de variáveis instrumentais $Z$, além de sofrer desvios aleatórios do vetor de perturbação estocástica - $V(U)$. 
A variável escalar $U$ captura todos os fatores não observados que afetam a equação estrutural. Desta forma, as condições prevalecentes em (2a) e (2c) mostram que o evento $\left\{Y \leq S_{\tau}(Y \mid X)\right\}$ é equivalente a $\{U \leq \tau\}$. Esta equivalência de eventos sugere uma reformulação na função quantil condicional: $S_{\tau}(Y \mid D, X)=D^{\prime} \alpha(\tau)+X^{\prime} \beta(\tau)$, em outras palavras: $P\left[Y \leq S_{\tau}(Y \mid D, X) \mid X, Z\right]=\tau$.

A função quantílica estrutural $S_{\tau}(Y \mid D, X)$ exibe características bastante diferentes da função tradicional $Q_{\tau}(Y \mid X)$. Fixando a variável endógena $D=$ ", a função $S_{\tau}(Y \mid D, X)$ descreve uma função quantílica de uma variável latente, por meio de uma perturbação aleatória em $U \sim U(0,1)$ e nos demais regressores exógenos. Contudo, a noção do contexto "aleatório" é violada em decorrência da relação entre $D$ e $U$, por meio da perturbação estocástica $V(U)$.

Ao invés disso, o modelo precisa incorporar a determinação da variável endógena por meio da equação (2b). Isso equivale a estimar $S_{\tau}(Y \mid d, X)$ por meio da técnica de variáveis instrumentais, tomando variações induzidas nos regressores endógenos por intermédio dos instrumentos $Z$, a fim de isolar as influências da variável escalar $U$ nos regressores da equação estrutural.

A partir da equação de identificação $P\left[Y \leq S_{\tau}(Y \mid D, X) \mid X, Z\right]=\tau$, pode-se estimar o vetor de parâmetros $\theta(\tau) \equiv[\alpha(\tau), \beta(\tau)]$ através da solução do problema:

$$
\begin{aligned}
& Q_{n}(\tau, \alpha, \beta, \gamma)=\sum_{i=1}^{n} \rho_{\tau}\left(y_{i}-S_{\tau}\left(Y=y \mid d_{i}, x_{i}\right)-f\left(x_{i}, z_{i}\right)^{\prime} \gamma\right) V_{i} \\
& \arg \min _{\beta, \gamma} Q_{n}(\tau, \alpha, \beta, \gamma)
\end{aligned}
$$

A função $\rho_{\tau}=u(\tau-1[u<0])$ representa a tradicional função-check dos modelos de regressão quantílica. Já $V_{i} \equiv V_{i}(\tau, x, z)$ consiste em uma função-escalar de "pesos", que por simplicidade e sem perdas de generalidade toma-se um valor. A funçãorepresenta uma projeção de mínimos quadrados da variável endógena $d$ nos regressores exógenos $x$ e nos instrumentos $z$. Adotando o algoritmo proposto por Chernozhukov e Hansen $(2006,2008)$, a função $f()$ será substituída pelo vetor de instrumentos $z$, assim como a função-escalar de pesos $V=1$.

\subsection{ALGORITMO DE ESTIMAÇÃO}

Para um dado valor do $\tau$-ésimo quantil, obtém-se uma estimativa dos parâmetros $[\tilde{\beta}(\alpha(\tau)), \tilde{\gamma}(\alpha(\tau))]$ :

$$
[\tilde{\beta}(\alpha(\tau)), \tilde{\gamma}(\alpha(\tau))] \equiv \arg \min _{\beta, \gamma} Q_{n}(\tau, \alpha, \beta, \gamma)
$$


Tomando um valor para $\tau$, o algoritmo procede na busca por um $\alpha(\tau)$ de forma que sua estimativa $\hat{\alpha}(\tau)$ conduza a um valor em $\tilde{\gamma}(\alpha(\tau))$ tão próximo de zero quanto for possível. Assim, isto é procedido da seguinte maneira:

$$
\hat{a}(\tau)=\arg \inf _{\alpha(\tau), \alpha \in \Lambda} n[\tilde{\gamma}(\alpha(\tau))]^{\prime} \hat{A}(\alpha)[\tilde{\gamma}(\alpha(\tau))]
$$

Em (4), $\Lambda$ corresponde ao espaço de parâmetros para $\alpha$ e $\hat{A}(\alpha)=A(\alpha)+O_{p}(1)$, em que $A(\alpha)$ corresponde a uma matriz definida-positiva e inversa da matriz de covariância de $\tilde{\gamma}(\alpha(\tau))-\sqrt{n}[\gamma(\alpha(\tau))-\tilde{\gamma}(\alpha(\tau))]$. A partir disso, o estimador de Regressão Quantílica por Variáveis Instrumentais (RQ-VI), $\hat{\theta}(\tau)$ é obtido aplicando o estimador de regressão quantílica de Koenker e Bassett (1978) de $F \equiv y_{i}-d_{i}{ }_{i} \hat{\alpha}(U)$ como função de $\left[x_{i}^{\prime}, z_{i}^{\prime}\right]$ :

$$
\hat{\theta}(\tau) \equiv[\hat{\alpha}(\tau), \tilde{\beta}(\tau)]
$$

De posse destes resultados, Chernozhukov, Hansen e Janssom (2009) provaram que, para um dado $\tau$-ésimo quantil, $\hat{\alpha}(\tau) \stackrel{p}{\rightarrow} \alpha(\tau), \tilde{\beta}(\tau) \stackrel{p}{\rightarrow} \beta(\tau)$ e $\tilde{\gamma}(\hat{\alpha}(\tau)) \stackrel{p}{\rightarrow} 0$, uma vez que a função quantílica estrutural é definida por $S_{\tau}(Y=y \mid d, x)=d^{\prime} \alpha(\tau)+x^{\prime} \beta(\tau)$, consequentemente, as estimativas convergem em probabilidade para os verdadeiros valores.

\subsection{MODELO ESTIMADO}

A partir do método de RQ-VI, o modelo estimado consiste na seguinte equação estrutural:

$$
\begin{aligned}
& \ln \left(y_{i}\right)=\beta_{0}+\beta_{1} \ln (P \& D)+\beta_{2} \ln (\text { Capex })+\beta_{3} \ln (\text { Emp })+\delta_{c}+\mu_{s}+\varepsilon_{i} \\
& \ln (P \& D)=\varphi\left(X, \ln \left(\pi_{i}\right), g_{\pi-3 \text { years }}, g_{y-3 \text { years }}, \tau, \vartheta\right)
\end{aligned}
$$

Conforme a equação estrutural (6a), as variáveis $y$, P\&D, Capex, Emp, correspondem, respectivamente, ao volume de vendas, aos investimentos em pesquisa e desenvolvimento, aos investimentos em bens de capital e ao número de funcionários das i-firmas. A equação (6b) corresponde ao primeiro estágio da estimação, levando em consideração os fatores que afetam a demanda por investimentos, incluindo os regressores exógenos $X=\left[\ln (P \& D, \ln (\right.$ Capex $)]$ e os instrumentos $Z=\left[\ln \left(\pi_{i}\right), g_{\pi-3_{\text {years }}}, g_{y-3 \text { years }}\right]$ que re- 
presentam, respectivamente $\left(\pi, g_{\pi}, g_{y}\right)$, aos níveis de lucros, taxa de crescimento dos lucros nos últimos três anos e a taxa de crescimento das vendas nos últimos três anos ${ }^{2}$.

O Quadro 1 apresenta uma síntese com a descrição das variáveis:

\section{Quadro 1 - Descrição das variáveis}

\begin{tabular}{lcc}
\hline Variável & Descrição da variável & Referências \\
\hline y & Volume de vendas & Montresor e Vezzani (2015) \\
P\&D & Investimentos em P\&D & Castellani et al. (2017), Montresor e Vezzani (2015), \\
Capex & Investimentos em bens de capital & Castellani et al. (2017) e Montresor e Vezzani (2015) \\
Emp & Estoque de funcionários & Bogliacino (2014) \\
$\pi$ & Volume de lucros brutos & Bogliacino e Pianta (2010) \\
g $\pi$ & Taxa de crescimento dos lucros & Aghion et al. (2012), Aghion, Howitt e Prantl (2015) \\
gy & Taxa de crescimento das vendas & Pindado, Queiroz e la Torre (2010), Lee e Choi (2015) \\
\hline
\end{tabular}

Fonte: Elaboração própria.

Por último, os vetores $\delta_{c}, \mu_{s}$ capturam os efeitos fixos relativos aos países e aos setores que afetam a dinâmica dos investimentos na variável resultado. As variáveis $\varepsilon, \vartheta$ representam a todos os demais fatores não observados e que exercem a mínima influência no modelo estimado. Para garantir os critérios de eficiência no modelo, as estimativas foram obtidas por meio da técnica de bootstrapping, desenvolvida por Efron (1979), tomando o número de réplicas como sendo igual a 400 conforme Andrews e Buchinsky (2001).

Juntamente ao método de regressão quantílica e regressão quantílica com variáveis instrumentais, foram empregados o método dos mínimos quadrados ordinários, as variáveis instrumentais e o método dos momentos generalizados, a fim de analisar os impactos sobre a média da distribuição e análise da validade e relevância dos instrumentos.

Para avaliar a importância dos instrumentos empregados foram adotados as estatísticas J-Hansen e o multiplicador lagrangeano (ML) de Kleibergen e Paap (2006).

2 Taxa de crescimento anual composta, em inglês compound anual growth rate (CAGR). A CAGR é expressa conforme a fórmula: $\left[\left(\frac{C}{B}\right)^{\frac{1}{t}}-1\right] * 100$. Na fórmula, $C$ representa a variável no ano corrente e $B$ a mesma no ano base. A variável " $\mathrm{t}$ " representa o número de período que, em questão, corresponde a $t=3$. Os valores são obtidos pelo relatório, que apresenta informações já calculadas das taxas de crescimento. 
Conforme Stock (2015) e Galvao e Montes-Rojas (2015), duas condições são fundamentais para a validade dos instrumentos: (1) os instrumentos precisam ser exógenos e, portanto, não correlacionados com a perturbação estocástica e; (2) a correlação parcial entre os instrumentos e a variável dependente deva ser elevada significativa. A junção das condições implica que o conjunto de instrumentos selecionados produz uma estimativa consistente dos parâmetros do modelo $\left(\hat{\beta}^{I V} \stackrel{p}{\rightarrow} \beta\right)$.

Tais condições são avaliadas por meio das duas estatísticas apresentadas. A estatística J-Hansen - para restrições de sobre-identificação - testa a hipótese nula de que os instrumentos não são correlacionados com a perturbação estocástica (PARENTE e SANTOS SILVA, 2012). Para verificar a relevância dos instrumentos foi aplicada a estatística do multiplicador lagrangeano (ML) de Kleibergen e Paap (2006), cuja hipótese nula implica nos instrumentos serem altamente correlacionados com as variáveis endógenas.

\subsection{ROBUSTEZ DOS INSTRUMENTOS}

Na literatura do crescimento endógeno, em especial nos recentes estudos de Aghion e Festré (2017), Aghion e Jaravel (2015), Aghion et al. (2012), Aghion, Howitt e Prantl (2015), têm apresentado que, nas condições de maximização dos lucros da firma monopolista, os lucros apropriados conduzem a novos investimentos em P\&D com o intuito de sustentar os esforços em inovação. Recentes estudos empíricos têm evidenciado esta relação, em destaque: Bogliacino e Cardona (2010), Bogliacino e Pianta (2010), Hall, Mairesse e Mohnen (2010), Pindado, Queiroz e la Torre (2010) e Lee e Choi (2015).

Conforme Bogliacino e Cardona (2010), a firma investe em pesquisa orientada por ganhos econômicos advindos da inovação, sejam por direitos de propriedade intelectual, defasagens no aprendizado, segredos industriais ou outras formas de proteção. Assim, a escolha do nível ótimo de investimento está condicionada aos lucros acumulados no tempo que aumentam a probabilidade de sucesso da inovação. Lee e Choi (2015) afirmaram que escalas crescentes de lucros associados a altas receitas indicam firmas bem-sucedidas, induzindo aumentos nos ativos de investimentos em inovação. Entretanto, escalas crescentes nos lucros revelam fundos internos que podem ser alocados nas atividades de pesquisa, sustentando os lucros futuros das empresas. Nesse caso, os autores classificam os lucros como um importante determinante dos investimentos alocados nas atividades em inovação.

Assim como os lucros, Pindado, Queiroz e La Torre (2010) apresentaram evidências empíricas, indicando que características das firmas a partir do desempenho -, como o crescimento das vendas dentre outros importantes fatores -, têm uma influência positiva nos investimentos em P\&D. Isso decorre desses investimentos e do alto crescimento das firmas para aumentar as oportunidades de expansão na escala dos 
lucros, transbordando em um efeito positivo no valor da empresa. Tal cenário foi diagnosticado por Lee e Choi (2015) ao analisar a influência da taxa de crescimento das vendas líquidas nos investimentos em P\&D de empresas do setor farmacêutico entre 2000 a 2012.

Assim como a literatura apresentada, a presente pesquisa empregará como instrumentos fatores relacionados com importantes características das firmas, apresentadas na Tabela 1:

Tabela 1 - Descrição dos instrumentos e testes aplicados

\begin{tabular}{cc}
\hline Instrumentos Excluídos & Instrumentos Incluídos \\
\hline $\ln (\pi)$ & $\ln ($ capex $)$ \\
$g_{\pi-3 y \text { years }}$ & $\ln (\mathrm{emp})$ \\
$\mathrm{g}_{\mathrm{y}-\text {-3years }}$ & Testes de validade dos instrumentos \\
\hline & Teste J-Hansen (HAYASHI, 2000) \\
HIPÓTESE NULA & HIPÓTESE ALTERNATIVA \\
\hline
\end{tabular}

Instr. válidos e não correlacionados com o erro Instr. inválidos e correlacionados com o erro

Teste de Kleibergen e Paap (2006)

HIPÓTESE NULA

HIPÓTESE ALTERNATIVA

Instrumentos não relevantes e fracamente ou não correlacionados com os regressores endógenos
Instrumentos relevantes e fortemente correlacionados com os regressores endógenos

Fonte: Elaboração própria.

A variável instrumentada consistiu no $\ln (\mathrm{P} \& \mathrm{D})$, enquanto os instrumentos excluídos consistiram no $\ln$ (lucros), $\mathrm{g}_{\text {lucros-3anos }}$ e $\mathrm{g}_{\text {vendas-3anos. }}$. Os instrumentos incluídos consistiram nos demais regressores exógenos. A estatística J-Hansen testa a validade dos instrumentos $(E(Z \mid \varepsilon)=0)$, em que $Z$ corresponde ao conjunto de instrumentos excluídos e incluídos. O teste do multiplicador lagrangeano (ML) de Kleibergen e Paap (2006) testa a relevância dos instrumentos excluídos $\left(Z^{*}\right)$ por meio da $\operatorname{cov}\left(Z^{*}, y_{-i}\right)=0$, em que $y_{-i}$ consiste no vetor de variáveis endógenas que, neste caso, representa $\ln (P \mho D)$. Logo, sob a hipótese nula do teste, a equação estrutural é subidentificada e, consequentemente, os instrumentos são redundantes (a condição de posto falha). 


\section{ANÁLISE DOS RESULTADOS}

\subsection{ANÁLISE DESCRITIVA DOS DADOS}

As principais estatísticas descritivas são apresentadas na Tabela 2, destacando os percentis $(25 \%, 50 \%, 75 \%$ e $95 \%)$, a média, o desvio-padrão e o coeficiente de variação (CV):

Tabela 2 - Estatísticas descritivas da amostra, ano 2012

\begin{tabular}{|c|c|c|c|c|c|c|c|}
\hline \multirow{2}{*}{ Variáveis } & \multicolumn{4}{|c|}{ Percentil } & \multirow{2}{*}{ Média } & \multirow{2}{*}{$\begin{array}{l}\text { Desvio- } \\
\text { Padrão }\end{array}$} & \multirow{2}{*}{$\mathrm{CV}$} \\
\hline & $25 \%$ & $50 \%$ & $75 \%$ & $95 \%$ & & & \\
\hline Inv. P\&D (€ Milhões) & 37,80 & 76,00 & 193,00 & $1.379,40$ & 320,45 & 763,80 & $238 \%$ \\
\hline Vendas (€ Milhões) & 961,10 & $2.446,85$ & $7.723,60$ & $43.198,00$ & $10.227,40$ & $23.437,00$ & $229 \%$ \\
\hline Inv. Capex (€ Milhões) & 37,70 & 105,15 & 378,30 & $2.803,70$ & 742,24 & $2.278,00$ & $307 \%$ \\
\hline Lucros (€ Milhões) & 81,40 & 220,35 & 710,20 & $4.833,50$ & $1.153,51$ & $3.040,00$ & $264 \%$ \\
\hline $\mathrm{G}_{\text {lucros }}(\%)$ & $-2,85$ & 10,25 & 28,10 & 85,50 & 17,12 & 47,21 & $276 \%$ \\
\hline $\mathrm{G}_{\text {vendas }}(\%)$ & 2,40 & 8,00 & 16,30 & 43,20 & 12,90 & 41,13 & $319 \%$ \\
\hline No Func. & $4.066,00$ & $10.166,50$ & $30.190,00$ & $128.705,00$ & $31.486,05$ & $55.996,00$ & $178 \%$ \\
\hline
\end{tabular}

Fonte: Elaboração própria.

Conforme os dados da tabela, é bastante perceptível que há distorções entre os diferentes percentis na distribuição das variáveis. Com relação aos investimentos em $\mathrm{P} \& \mathrm{D}$, a cauda inferior representada pelo percentil menor (25\%) revela um baixo volume nos investimentos, que vai aumentando gradativamente junto com os percentis superiores. Nesse sentido, $25 \%$ das firmas da amostra apresentam um volume de investimentos não superior a $€ 37,8$ milhões. Trata-se de um resultado bem abaixo da média e bastante inferior, se comparado com o percentil 95\%, cujas firmas apresentam um volume de investimentos não superior a $€ 1.379,40$ milhões.

Considerando o nível de vendas das firmas, o mesmo padrão é também observado. Dessa forma, $25 \%$ das firmas administram um nível de vendas de até no máximo $€$ 961,10 milhões, bem abaixo da cauda superior da distribuição, na qual 95\% das firmas passam a desfrutar de um nível de vendas não superior a $€ 43.198$ milhões. Esse padrão mostra o aspecto heterogêneo da amostra que fragiliza as análises pautadas na média da distribuição, o que pode ser percebido pelos coeficientes de variação bastante elevados.

No tocante às taxas de crescimento (vendas e lucros), o padrão de evolução na taxa acompanha os percentis analisados, mostrando uma ampla distorção nas taxas e a imprecisão da média frente à grande dispersão da amostra. Os coeficientes analisados revelam que a taxa de crescimento nos lucros apresentou o maior nível de imprecisão 
a partir da sua média, seguidos dos investimentos em bens de capital e, por último, do número de funcionários.

Tal anomalia corrobora com a imprecisão das técnicas tradicionais de regressão, destacando as vantagens da abordagem quantílica que passa a centrar a análise a partir de diferentes pontos ou "faixas" da distribuição.

A Tabela 3 mostra os coeficientes de correlação e os níveis de significância para o teste de hipótese $H_{0}: \rho_{i, j}=0$.

Tabela 3 - Matriz de correlação e significância estatística, ano 2012

\begin{tabular}{lccccccc}
\hline & Inv. P\&D & Vendas & Inv. Capex & Lucros & No Func. & Glucros & Gvendas \\
\hline Inv. P\&D & 1,0000 & - & - & - & - & - \\
Vendas & $0,4237^{* * *}$ & 1,0000 & - & - & - & - & - \\
Inv. Capex & $0,3070^{* * *}$ & $0,8596^{* * *}$ & 1,0000 & - & - & - & - \\
Lucros & $0,4351^{* * *}$ & $0,8013^{* * *}$ & $0,8184^{* * *}$ & 1,0000 & - & - & - \\
No Func. & $0,4665^{* * *}$ & $0,5897^{* * *}$ & $0,5080^{* * *}$ & $0,4277^{* * *}$ & 1,0000 & - & - \\
$\mathbf{G}_{\text {lucros }}$ & $0,0487^{*}$ & 0,0211 & 0,0092 & 0,0296 & 0,0017 & 1,0000 & 1,0000 \\
$\mathbf{G}_{\text {vendas }}$ & $-0,0290$ & $-0,0220$ & $-0,0122$ & $-0,0047$ & $-0,0407^{*}$ & $0,1009^{* * *}$ & 1, \\
\hline
\end{tabular}

Nota: $\left(^{* * *}\right) p<0,01 ;(* *) p<0,05 ;\left({ }^{*}\right) p<0,1$.

Fonte: Elaboração própria.

De acordo com os resultados apresentados na tabela, os coeficientes de correlação para as variáveis em nível apresentaram sinais positivos e estatisticamente significativos ao nível de $1 \%$. Isso aponta para a rejeição da hipótese nula de coeficientes estatisticamente nulos ou com nenhuma associação linear entre as variáveis, mesmo considerando um nível mínimo de significância de $1 \%$.

Embora se perceba uma forte significância estatística, o mesmo não pode ser observado ao considerar o cruzamento com as variáveis taxas de crescimento. Com relação à taxa de crescimento nos lucros, somente com os níveis de investimento em P\&D e com o crescimento das vendas apresentou-se associação linear positiva significativa. Doravante ao crescimento nos lucros, o tamanho das firmas, medido pelo número de funcionários, apresentou uma associação linear negativa e significativa.

Ainda que as taxas de crescimento venham a apresentar baixos padrões de associação linear com as variáveis, cabe ressaltar que as distorções de escala numérica apresentadas nas demais variáveis possam contribuir para reduzir esta associação. Para capturar mais precisamente as relações entre as variáveis, transformações monotônicas (como aplicações de logaritmo natural) consistem em um refúgio essencial para a análise do modelo a seguir. 


\subsection{RESULTADOS DO MODELO}

Nesta seção, serão apresentados os resultados do modelo (6a), a partir das técnicas de regressão quantílica (RQ) e regressão quantílica com variáveis instrumentais (RQ-VI). Porém, os resultados apresentados na Tabela 4 correspondem às estimativas do modelo obtidas a partir de três técnicas diferentes: variáveis instrumentais (VI), método dos momentos generalizados (MMG) e mínimos quadrados ordinários (MQO).

\section{Tabela 4 - Resultados do Modelo (6a), ano 2012}

\begin{tabular}{lccc}
\hline VARIÁVEIS & $(1)$ & $(2)$ & $(3)$ \\
& VI & MMG & MQO \\
\hline $\log ($ P\&D $)$ & $1.049^{* * *}$ & & \\
& $(0.0929)$ & $1.037^{* * *}$ & $0.249^{* * *}$ \\
$\log ($ Capex $)$ & 0.0627 & $(0.0927)$ & $(0.0174)$ \\
& $(0.0405)$ & 0.0628 & $0.328^{* * *}$ \\
$\log ($ Emp) & $0.158^{* * *}$ & $(0.0405)$ & $(0.0212)$ \\
& $(0.0435)$ & $0.168^{* * *}$ & $0.413^{* * *}$ \\
Constante & $2.903^{* * *}$ & $(0.0431)$ & $(0.0275)$ \\
& $(0.282)$ & $2.846^{* * *}$ & $1.895^{* * *}$ \\
& & $(0.280)$ & $(0.201)$ \\
\hline Amostra & 2000 & 2000 & 2000 \\
$\mathrm{R}^{2}$ & 0.710 & 0.715 & 0.895 \\
Kleibergen-Paaptest & $89.754^{* * *}$ & $89.755^{* * *}$ & - \\
Hansen-J statistic & 3.876 & 3.776 & - \\
\hline Ef.Fixos-País & SIM & SIM & SIM \\
Ef.Fixos-Setor & SIM & & \\
\hline
\end{tabular}

Nota: $\left.\left({ }^{* *}\right) p<0.01 ;(* *) p<0.05 ;{ }^{*}\right) p<0.1$. As estimativas de erro-padrão foram corrigidas a partir do método de bootstrap usando 400 réplicas.

Fonte: Elaboração própria.

Os resultados do modelo sugerem haver uma clara distorção nas elasticidades calculadas a partir dos métodos de VI (coluna 1) e MMG (coluna 2). Comparando o estimador tradicional MQO (coluna 3) com as técnicas alternativas, as elasticidades calculadas apresentam disparidades de quatro vezes maior em relação ao parâmetro tendencioso (significativos ao nível de 1\%). Isso revela uma subestimação do impacto 
dos investimentos em P\&D nas vendas das firmas, ao contrário dos investimentos em bens de capital. Ao contrário disso, as estimativas por MQO revelaram uma clara tendenciosidade ao superestimar o impacto dos investimentos em bens de capital. Tal padrão é contrariado pelas estimativas de VI e MMG, que apresentaram elasticidades proporcionalmente inferiores e equivalente a 19\% do parâmetro em MQO (embora não significativo ao nível máximo de 10\%).

De todos os parâmetros estimados, a elasticidade da mão de obra apresentou maior valor no método MQO, deslocando o impacto no aumento das vendas em maior grandeza para este insumo. Comparando as elasticidades dos demais insumos no método MQO, nota-se que as elasticidades de P\&D e Capex são equivalentes a 60\% e 79\% da elasticidade da mão de obra, respectivamente. Esse aspecto é revertido nos métodos de VI e MMG, mostrando a importância relativa dos investimentos em P\&D sobre os demais insumos.

No tocante aos testes de validade e relevância dos instrumentos, as estatísticas J-Hansen e ML de Kleibergen e Paap (2006) demonstraram a viabilidade dos instrumentos empregados. Primeiramente, o teste J-Hansen demonstrou não rejeitar a hipótese nula ao nível máximo 10\%, sinalizando que os instrumentos (incluídos e excluídos) não são estatisticamente correlacionados com a perturbação estocástica. Em segundo, fica evidente pela significância do teste de Kleibergen e Paap (2006) que os instrumentos são significativamente correlacionados com os regressores endógenos, o que ajuda a capturar maior informação desta variável sem os riscos de uma nova endogeneidade.

Em seguida, a Tabela 5 apresenta o mesmo modelo estimado a partir das técnicas selecionadas com uma restrição: selecionando somente as firmas atuantes nos setores intensivos em alta e média-alta tecnologia, excluindo os efeitos fixos na estimação.

Conforme os resultados da tabela, a elasticidade dos investimentos em P\&D apresenta um valor subestimado a partir do método MQO. As estimativas por VI e MMG apresentaram, respectivamente, uma magnitude de aproximadamente 4,5 vezes maior em relação ao parâmetro de MQO (significativo a 1\%). Assim, 1\% no aumento destes investimentos contribui em média para um crescimento nas vendas de aproximadamente $0,78 \%$ (VI) e 0,77\% (MMG), contra 0,17\% (MQO).

Com relação aos investimentos em bens de capital, nota-se uma queda nas elasticidades comparando-se os métodos VI e MMG com MQO. Um aumento de $1 \%$ nos investimentos gera um crescimento médio de $0,10 \%$ (VI) e $0,11 \%$ (MMG), contra 0,31\% (MQO) - parâmetros significativos ao nível de 1\%. Essa subestimação corresponde a uma queda no valor das estimativas de aproximadamente 68\% (VI) e 69\% (MMG) em comparação ao estimador de MQO - parâmetros significativos ao nível de $1 \%$.

O mesmo padrão de subestimação pode ser visto na elasticidade do estoque de mão de obra. Observa-se uma redução de aproximadamente 41\% (VI) e 40\% (MMG) 
em comparação com a estimativa de MQO - parâmetros significativos ao nível de $1 \%$. Neste sentido, um aumento de $1 \%$ na contratação de mão de obra contribui para um crescimento médio nas vendas de aproximadamente 0,34\% (VI), 0,35\% (MMG) e $0,59 \%(\mathrm{MQO})$.

\section{Tabela 5 - Resultados do Modelo (6a) por setores intensivos em alta tecnologia, ano 2012}

\begin{tabular}{|c|c|c|c|}
\hline \multirow{2}{*}{ VARIÁVEIS } & (1) & (2) & (3) \\
\hline & VI & MMG & MQO \\
\hline \multirow[t]{2}{*}{$\log (\mathrm{P} \& \mathrm{D})$} & $0.786^{* * *}$ & $0.771^{\star * *}$ & $0.174^{* * *}$ \\
\hline & $(0.0635)$ & $(0.0631)$ & $(0.0219)$ \\
\hline \multirow[t]{2}{*}{$\log ($ Capex $)$} & $0.0991^{* * *}$ & $0.105^{\star * *}$ & $0.310^{* * *}$ \\
\hline & $(0.0350)$ & $(0.0348)$ & $(0.0308)$ \\
\hline \multirow[t]{2}{*}{$\log (\mathrm{Emp})$} & $0.348^{* * *}$ & $0.351^{* * *}$ & $0.589^{* * *}$ \\
\hline & $(0.0314)$ & $(0.0314)$ & $(0.0361)$ \\
\hline \multirow[t]{2}{*}{ Constante } & $0.417^{\star *}$ & $0.443^{* *}$ & 0.0477 \\
\hline & $(0.199)$ & $(0.198)$ & $(0.220)$ \\
\hline Amostra & 967 & 967 & 967 \\
\hline $\mathrm{R}^{2}$ & 0.746 & 0.753 & 0.858 \\
\hline Kleibergen-Paaptest & $110.009^{* * *}$ & $110.029^{* * *}$ & - \\
\hline Hansen-J statistic & 4.550 & 4.504 & - \\
\hline Ef.Fixos-País & - & - & - \\
\hline Ef.Fixos-Setor & - & - & - \\
\hline
\end{tabular}

Fonte: Elaboração própria.

Nota: $\left.\left(^{* *}\right) p<0.01 ;{ }^{* *}\right) p<0.05 ;$ e $\left.{ }^{*}\right) p<0.1$. As estimativas de erro-padrão foram corrigidas a partir do método de bootstrap usando 400 réplicas. Os setores intensivos em alta e média-alta tecnologia correspondem a: pharmaceuticals \& biotechnology; health care equipment \& services; technology hardware \&equipment; software \& computer services and aerospace \& defence; electronics \& electrical equipment; automobiles \& parts; industrial engineering \& machinery; chemicals; personal goods; household goods; general industrials; support services.

Em seguida, os instrumentos apresentam os mesmos atributos de validade e relevância. A estatística J-Hansen demonstra que os instrumentos são não correlacionados com a perturbação estocástica, sinalizando para uma clara validade. Além disso, o teste de Kleibergen e Paap (2006) confirma a relevância dos instrumentos ao rejeitar a hipótese nula ao nível de $1 \%$. 
Embora o modelo estimado para os setores de alta tecnologia apresente uma significativa associação entre as variáveis, não se pode deixar de destacar a queda visível nas elasticidades dos investimentos em P\&D para este grupo de firmas. Uma possível explicação para este fenômeno pode ser atribuída à exclusão dos efeitos fixos dos setores e países na estimação. Dessa forma, características particulares entre setores podem conduzir a estratégias mais ou menos intensivas nos investimentos, assim como o ambiente institucional em cada país. Nesta ordem de fatores, informações significativas e excluídas na perturbação estocástica podem estar correlacionadas com os regressores, conduzindo a estimativas tendenciosas dos parâmetros.

A partir disso, a Tabela 6 passa a incluir os efeitos fixos no modelo (6a) para os setores intensivos em alta e média-alta tecnologia.

\section{Tabela 6 - Resultados do Modelo (6a) por setores intensivos em alta tecnologia, ano 2012}

\begin{tabular}{|c|c|c|c|}
\hline \multirow{2}{*}{ VARIÁVEIS } & (1) & (2) & (3) \\
\hline & VI & MMG & MQO \\
\hline \multirow[t]{2}{*}{$\log (\mathrm{P} \& \mathrm{D})$} & $0.961^{\star * *}$ & $0.951^{\star * *}$ & $0.188^{\star * *}$ \\
\hline & $(0.0819)$ & $(0.0816)$ & $(0.0253)$ \\
\hline \multirow[t]{2}{*}{$\log ($ Capex $)$} & 0.0153 & 0.0180 & $0.282^{* * *}$ \\
\hline & $(0.0406)$ & $(0.0406)$ & $(0.0306)$ \\
\hline \multirow[t]{2}{*}{$\log (E m p)$} & $0.244^{* * *}$ & $0.248^{\star * *}$ & $0.598^{* * *}$ \\
\hline & $(0.0412)$ & $(0.0411)$ & $(0.0403)$ \\
\hline \multirow[t]{2}{*}{ Constante } & $1.515^{* * *}$ & $1.491^{* * *}$ & -0.0357 \\
\hline & $(0.246)$ & $(0.246)$ & $(0.273)$ \\
\hline Amostra & 967 & 967 & 967 \\
\hline $\mathrm{R}^{2}$ & 0.732 & 0.737 & 0.863 \\
\hline Kleibergen-Paaptest & $88.900^{* * *}$ & $89.993^{* * *}$ & - \\
\hline Hansen-J statistic & 3.725 & 3.625 & - \\
\hline Ef.Fixos-País & SIM & SIM & SIM \\
\hline Ef.Fixos-Setor & SIM & SIM & SIM \\
\hline
\end{tabular}

Fonte: Elaboração própria.

Nota: $\left.\left(^{* * *}\right) p<0.01 ;\left(^{* *}\right) p<0.05 ;{ }^{*}{ }^{*}\right) p<0.1$. As estimativas de erro-padrão foram corrigidas a partir do método de bootstrap usando 400 réplicas. Os setores intensivos em alta e média-alta tecnologia correspondem a: pharmaceuticals \& biotechnology; health care equipment \& services; technology hardware \& equipment; software \& computer services and aerospace \& defence; electronics \& electrical equipment; automobiles \& parts; industrial engineering \& machinery; chemicals; personal goods; household goods; general industrials; support services. 
De acordo com as informações da tabela, a inclusão dos efeitos fixos afeta diretamente a magnitude das elasticidades estimadas. Nota-se que as elasticidades dos investimentos em P\&D apresentam valores bem acima dos apresentados quando excluímos os efeitos dos setores e países. Logo, um aumento de $1 \%$ nos investimentos em P\&D repercute em um crescimento médio nas vendas de 0,96\% (VI), 0,95\% (MMG) e 0,19\% (MQO). Apesar das elasticidades apresentarem uma magnitude inferior em relação à amostra total (métodos VI e MMG), o distanciamento em relação ao parâmetro por MQO aumentou, revelando uma dimensão de aproximadamente cinco vezes maior para ambos os parâmetros.

No tocante aos investimentos em bens de capital, as elasticidades apresentaram não significância estatística, ainda que os sinais esperados apresentassem coerentes e com o mesmo padrão de redução ao se comparar com as estimativas por MQO. A redução foi de aproximadamente $95 \%$ e $94 \%$ para os métodos VI e MMG, respectivamente, em comparação com o método MQO.

As elasticidades da mão de obra apresentaram uma queda para os métodos VI e MMG após a inclusão dos efeitos fixos. Isto novamente sinaliza a perda da importância relativa deste insumo nos setores intensivos em tecnologia.

Com um aumento de $1 \%$ no insumo força de trabalho, espera-se um crescimento médio nas vendas de aproximadamente 0,24\% (VI), 0,25\% (MMG) e 0,60\% (MQO). A redução relativa das elasticidades para os métodos VI e MMG em relação ao MQO foi, respectivamente, de 59\% para ambos os métodos. Estes resultados apontam para a fragilidade do método MQO que superestima a elasticidade do insumo trabalho em relação aos investimentos em P\&D. Ainda que este insumo tenha sua importância na dinâmica da firma, as estimativas de VI e MMG revelam que, estrategicamente, os investimentos em P\&D são mais responsáveis pelo deslocamento da fronteira, o que explicaria as diferenças nas elasticidades em P\&D entre os métodos.

As estatísticas referentes à validade e relevância dos instrumentos satisfazem os critérios de elegibilidade. O teste J-Hansen não rejeita a hipótese nula dos instrumentos serem não correlacionados com a perturbação estocástica, mesmo considerando o nível máximo de significância (10\%).

A estatística de Kleibergen e Paap (2006) apresenta rejeitar a hipótese nula ao nível de 1\% (VI e MMG). Desta forma, os instrumentos excluídos apresentam uma covariância significativa com os regressores endógenos, sendo, portanto, relevante para explicá-los. Neste sentido, a utilização de instrumentos "fracamente" correlacionados aumenta o viés nos coeficientes VI/MMG estimados, piorando as aproximações de grandes amostras a partir das distribuições de amostras finitas (KLEIBERGEN e PAAP, 2006).

A Tabela 7 apresenta os resultados do modelo, confrontando o estimador tradicional de regressão quantílica com o método alternativo de variáveis instrumentais. 
Tabela 7 - Resultados do Modelo (6a) a partir do método de regressão quantílica - amostra total, ano 2012

\begin{tabular}{|c|c|c|c|c|c|c|c|c|}
\hline \multirow{4}{*}{ VARIÁVEIS } & \multicolumn{8}{|c|}{ Quantil } \\
\hline & \multicolumn{2}{|c|}{$25 \%$} & \multicolumn{2}{|c|}{$50 \%$} & \multicolumn{2}{|c|}{$75 \%$} & \multicolumn{2}{|c|}{$95 \%$} \\
\hline & (1) & (2) & (3) & (4) & (5) & (6) & (7) & (8) \\
\hline & RQ-VI & RQ & RQ-VI & RQ & RQ-VI & RQ & RQ-VI & RQ \\
\hline \multirow[t]{2}{*}{$\log (\mathrm{P} \& \mathrm{D})$} & $0.949^{* * *}$ & $0.295^{\star * *}$ & $1.205^{\star * *}$ & $0.231^{* * *}$ & $1.312^{* * *}$ & $0.217^{* * *}$ & $1.668^{* * *}$ & $0.129^{* *}$ \\
\hline & $(0.126)$ & $(0.0173)$ & $(0.145)$ & $(0.0146)$ & $(0.168)$ & $(0.0248)$ & $(0.369)$ & $(0.0599)$ \\
\hline \multirow[t]{2}{*}{$\log ($ Capex $)$} & 0.0365 & $0.274^{* * *}$ & 0.0400 & $0.305^{* * *}$ & 0.0611 & $0.313^{* * *}$ & 0.117 & $0.408^{* * *}$ \\
\hline & $(0.0512)$ & $(0.0174)$ & $(0.0574)$ & $(0.0156)$ & $(0.0683)$ & $(0.0213)$ & $(0.150)$ & $(0.0514)$ \\
\hline \multirow[t]{2}{*}{$\log (E m p)$} & $0.184^{* * *}$ & $0.467^{\star * *}$ & 0.0687 & $0.459^{* * *}$ & $0.141^{\star *}$ & $0.427^{* * *}$ & 0.170 & $0.357^{* * *}$ \\
\hline & $(0.0535)$ & $(0.0194)$ & $(0.0671)$ & $(0.0167)$ & $(0.0714)$ & $(0.0247)$ & $(0.157)$ & $(0.0597)$ \\
\hline \multirow[t]{2}{*}{ Constante } & $2.578^{* * *}$ & $0.755^{* * *}$ & $3.436^{* * *}$ & $1.457^{* * *}$ & $2.588^{* * *}$ & $2.453^{* * *}$ & $1.989^{* *}$ & $4.295^{* * *}$ \\
\hline & $(0.328)$ & $(0.176)$ & $(0.419)$ & $(0.155)$ & $(0.438)$ & $(0.192)$ & $(0.962)$ & $(0.464)$ \\
\hline Amostra & 2,000 & 2,000 & 2,000 & 2,000 & 2,000 & 2,000 & 2,000 & 2,000 \\
\hline Ef.Fixos-País & SIM & SIM & SIM & SIM & SIM & SIM & SIM & SIM \\
\hline Ef.Fixos-Setor & SIM & SIM & SIM & SIM & SIM & SIM & SIM & SIM \\
\hline
\end{tabular}

Fonte: Elaboração própria.

Nota: $\left.\left(^{* *}\right) p<0.01 ;{ }^{* *}\right) p<0.05 ;$ e $\left.{ }^{*}\right) p<0.1$. As estimativas de erro-padrão foram corrigidas a partir do método de bootstrap usando 400 réplicas.

De acordo com os resultados apresentados, ficam evidentes as distorções entre as estimativas na medida em que se incorporam as influências dos lucros e do crescimento nas decisões de investimento. Analisando as elasticidades em P\&D nos diferentes quantis condicionais, o impacto dos investimentos nas vendas aumenta na medida em que os quantis crescem, sugerindo que as empresas com maior volume nas vendas apresentam maior retorno ou, possivelmente, maior apropriação no uso destes investimentos (RQ-VI). Este padrão apresenta-se oposto ao método tradicional RQ, cujas elasticidades estimadas decrescem com o aumento do quantil - significativos a $1 \%$. $\mathrm{O}$ Gráfico 1 apresenta de forma resumida a evolução das elasticidades ao longo dos diferentes quantis condicionais. 
Gráfico 1 - Evolução das elasticidades dos investimentos em P\&D por quantil, ano 2012

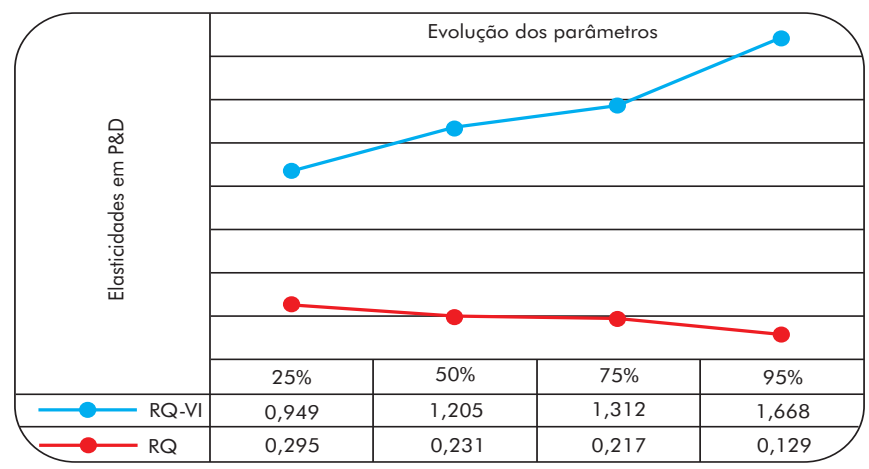

Fonte: Elaboração própria.

Além disso, o viés ocasionado pela exclusão dos instrumentos torna-se consideravelmente maior quando aumentamos os quantis. A estimativa de regressão quantílica por variáveis instrumentais é três vezes maior no primeiro quantil; cinco vezes maior no segundo; seis vezes maior no terceiro; e 12 vezes maior no último quantil. Esses resultados são apresentados no Gráfico 2, destacando o distanciamento entre as elasticidades em $\mathrm{P} \& \mathrm{D}$ em cada quantil.

$\mathrm{O}$ aumento do viés ao longo dos quantis sugere que as firmas com maior performance obtêm melhores resultados, de forma que a regressão quantílica tradicional incorre em erros de medida que penaliza as firmas com maior desempenho. Essa "dicotomia" mostra um padrão inverso da convergência tradicional, cujas firmas operantes na fronteira passam a crescer mais a partir de determinados investimentos em relação às firmas mais afastadas da fronteira.

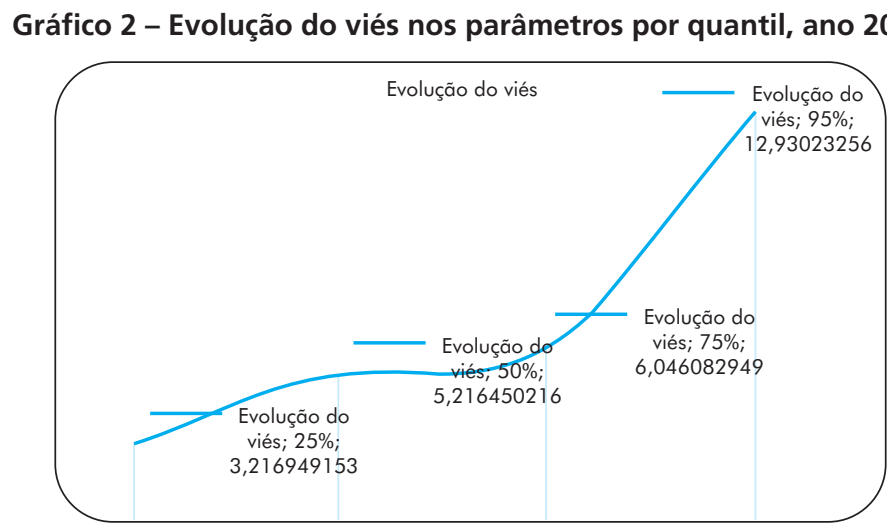

Nota: A medida de viés entre as estimativas por quantil é obtida a partir da razão Fonte: Elaboração própria. 
Além das distorções apresentadas, os resultados para os investimentos em bens de capital não se tornam tão divergentes, relativamente. As estimativas RQ revelam que na cauda inferior da distribuição condicional, a elasticidade estimada consiste em média $0,27 \%$, que aumenta gradativamente até atingir $0,41 \%$ no quantil superior, sugerindo que o impacto desses investimentos se torna maior nas firmas com maior desempenho.

Da mesma forma, as estimativas RQ-VI são coerentes com esta evolução, entretanto a magnitude estimada é notoriamente inferior ao longo de todos os quantis, variando de 0,04\% na cauda inferior da distribuição para 0,11\% no quantil 95\%. Comparando-se os limites dos quantis estimados, as estimativas de RQ-VI é $87 \%$ menor no quantil 25\% e $71 \%$ no quantil superior, $95 \%$. Ainda que os resultados sejam bastante diferentes, as estimativas de RQ-VI não apresentaram sinais de significância estatística.

Por último, as elasticidades do insumo mão de obra demonstraram uma tendência diferente, com estimativas decrescentes ao longo dos quantis. As estimativas RQ mostram que as elasticidades variaram de $0,46 \%$ no quantil $25 \%$ para $0,36 \%$ no quantil 95\%. Da mesma forma, as estimativas RQ-VI apresentaram uma tendência decrescente, oscilando de $0,18 \%$ na cauda inferior da distribuição para $0,17 \%$ no quantil superior. Porém, somente nos quantis $25 \%$ e $75 \%$ que as estimativas RQ-VI apresentaram significância estatística.

Conforme os resultados da Tabela 8, as elasticidades dos investimentos em P\&D apresentaram notórias diferenças entre os métodos. No método RQ tradicional, a elasticidade em P\&D evolui decrescentemente ao longo dos quantis condicionais. Um aumento de $1 \%$ nestes investimentos contribui para um crescimento médio nas vendas equivalente a $0,37 \%$ nas firmas com baixo volume de vendas (cauda inferior da distribuição) - significativo ao nível de $1 \%$. Considerando o mesmo quantil, o método RQ-VI estimou um coeficiente de elasticidade quase três vezes maior do que o método tradicional. Portanto, um aumento de $1 \%$ nos investimentos em P\&D nas firmas com baixa performance contribui para um crescimento médio nas vendas de aproximadamente $1,02 \%$ - significativo ao nível de $1 \%$.

Considerando as firmas situadas na mediana (quantil 50\%), o coeficiente de elasticidade passa a ser $8 \%$ maior em relação ao primeiro quantil. Logo, $1 \%$ de aumento dos investimentos contribui para um crescimento médio nas vendas de $1,10 \%$, bem acima do método tradicional RQ que corresponde a 0,34\% (significativo ao nível de $1 \%$ ).

Avaliando nos quantis superiores (75\% e 95\%), a elasticidade estimada torna-se ainda maior, correspondendo a 1,13\% e 1,55\%, respectivamente. Em direção contrária, o método RQ apresenta elasticidades que passam a serem menores nestes quantis, de $0,26 \%$ no quantil $75 \%$ e $0,17 \%$ no quantil $95 \%$, embora este último tenha se revelado não significativo. 
Outro destaque consiste nas elasticidades da mão de obra, que apresentaram resultados menores no método RQ-VI em relação ao RQ tradicional. Os coeficientes estimados apresentam valores decrescentes nos quantis, passando de $0,53 \%$ no primeiro quantil para $0,47 \%$ no último quantil - considerando o método RQ. Na abordagem alternativa (RQ-VI), os coeficientes estimados apresentam uma pequena oscilação com tendência crescente, passando de $0,22 \%$ no primeiro quantil para $0,27 \%$ no último quantil - parâmetros significativos a $1 \%$.

Novamente, percebe-se uma tendência de superestimação no coeficiente de elasticidade da mão de obra no método tradicional RQ. Da mesma maneira que apresentado na amostra total, as firmas atuantes nos setores intensivos em alta e média-alta tecnologia tendem a demonstrar maiores resultados no uso dos investimentos em P\&D em comparação com o insumo mão de obra.

Tabela 8 - Resultados do Modelo (6a) a partir do método de regressão
quantílica - setores de alta e média-alta tecnologia, ano 2012

\begin{tabular}{|c|c|c|c|c|c|c|c|c|}
\hline \multirow{4}{*}{ VARIÁVEIS } & \multicolumn{8}{|c|}{ Quantil } \\
\hline & \multicolumn{2}{|c|}{$25 \%$} & \multicolumn{2}{|c|}{$50 \%$} & \multicolumn{2}{|c|}{$75 \%$} & \multicolumn{2}{|c|}{$95 \%$} \\
\hline & (1) & (2) & (3) & (4) & (5) & (6) & (7) & (8) \\
\hline & RQ-VI & RQ & RQ-VI & RQ & RQ-VI & RQ & RQ-VI & RQ \\
\hline \multirow[t]{2}{*}{$\log (\mathrm{P} \& \mathrm{D})$} & $1.021^{* * *}$ & $0.375^{\star * *}$ & $1.103^{\star * *}$ & $0.342^{\star * *}$ & $1.127^{\star * *}$ & $0.263^{\star * *}$ & $1.549^{*}$ & 0.171 \\
\hline & $(0.0748)$ & $(0.0200)$ & $(0.130)$ & $(0.0253)$ & $(0.157)$ & $(0.0354)$ & $(0.830)$ & $(0.109)$ \\
\hline \multirow[t]{2}{*}{$\log ($ Capex $)$} & -0.0273 & $0.159^{* * *}$ & -0.0267 & $0.150^{* * *}$ & 0.0377 & $0.212^{* * *}$ & 0.0368 & $0.264^{* * *}$ \\
\hline & $(0.0487)$ & $(0.0244)$ & $(0.0551)$ & $(0.0258)$ & $(0.0565)$ & $(0.0358)$ & (0.119) & $(0.0494)$ \\
\hline \multirow[t]{2}{*}{$\log (E m p)$} & $0.218^{* * *}$ & $0.526^{\star * *}$ & $0.166^{\star * *}$ & $0.530^{* * *}$ & $0.201^{\star * *}$ & $0.509^{* * *}$ & $0.271^{\star * *}$ & $0.469^{* * *}$ \\
\hline & $(0.0487)$ & $(0.0234)$ & $(0.0632)$ & $(0.0287)$ & $(0.0648)$ & $(0.0377)$ & $(0.0845)$ & $(0.0983)$ \\
\hline \multirow[t]{2}{*}{ Constante } & $1.440^{* * *}$ & -0.249 & $1.909^{* * *}$ & 0.271 & $1.638^{* * *}$ & $0.957^{\star * *}$ & 0.282 & $2.792^{* * *}$ \\
\hline & $(0.312)$ & $(0.185)$ & $(0.368)$ & $(0.208)$ & $(0.433)$ & $(0.247)$ & (2.114) & $(0.550)$ \\
\hline Amostra & 967 & 967 & 967 & 967 & 967 & 967 & 967 & 967 \\
\hline Ef.Fixos-País & SIM & SIM & SIM & SIM & SIM & SIM & SIM & SIM \\
\hline Ef.Fixos-Setor & SIM & SIM & SIM & SIM & SIM & SIM & SIM & SIM \\
\hline
\end{tabular}

Nota: $\left.\left(^{* * *}\right) p<0.01 ;\left(^{* *}\right) p<0.05 ; e^{*}\right) p<0.1$. As estimativas de erro-padrão foram corrigidas a partir do método de Bootstrap usando 400 réplicas. Os setores intensivos em alta e média-alta tecnologia correspondem a: pharmaceuticals \&biotechnology; health care equipment \& services; technology hardware \&equipment; software \& computer services and aerospace \& defence; electronics \& electrical equipment; automobiles \& parts; industrial engineering \& machinery; chemicals; personal goods; household goods; general industrials; support services.

Fonte: Elaboração própria. 
Por último, as elasticidades dos investimentos em bens de capital apresentaram sinal negativo nos quantil $25 \%$ e na mediana. Entretanto, os coeficientes estimados não apresentaram sinais de significância estatística.

\section{DISCUSSÃO COM PESQUISAS RECENTES}

Os resultados apresentados mostram que diferentes contextos de performance afetam significativamente os resultados dos investimentos. Além disso, fatores que capturam o desempenho das firmas, como os lucros e as taxas de crescimento, afetam os resultados dos investimentos em P\&D, subestimando as elasticidades quando este vetor de influência é desconsiderando no modelo.

Um recente estudo de Montresor e Vezzani (2015) estimou uma função de produção que busca capturar as influências dos investimentos em $\mathrm{P} \& \mathrm{D}$, bens de capital e estoque de trabalho no desempenho das firmas. Por meio da técnica de regressão quantílica com dados em painel, os resultados do estudo apontaram para coeficientes de elasticidade em P\&D significativamente maiores nos quantis inferiores e gradativamente menores nos quantis superiores. O padrão decrescente nos resultados aponta para as mesmas conclusões: estimativas tendenciosas a partir da técnica tradicional que desconsidera os problemas associados à endogeneidade nas variáveis.

Dividindo o modelo estimado por grupos de intensidade tecnológica, o estudo de Montresor e Vezzani (2015) apresentou que o padrão decrescente nas elasticidades continua evidente entre os setores, com uma ênfase maior no impacto dos investimentos para os setores de alta tecnologia. Os coeficientes de elasticidade da mão de obra revelaram valores superiores em relação a $\mathrm{P} \& \mathrm{D}$, destacando a superestimação deste insumo na técnica tradicional RQ. Isso conduz a uma avaliação de política equivocada, sugerindo que a expansão do emprego, em modo geral, contribui para um crescimento mais rápido que os investimentos em $\mathrm{P} \& \mathrm{D}$.

Ao contrário disto, a técnica de RQ-VI aponta para resultados opostos, cuja expansão do insumo-trabalho, via áreas especificas na pesquisa, tende a apresentar melhores resultados. Isto decorre do fato apresentado por Hall, Lotti e Mairesse (2008, 2013) e Hall e Lerner (2009), em que osinvestimentos em P\&D apresentam um padrão distinto dos demais recursos: financiar um conhecimento tácito e incorporado nos funcionários de pesquisa, que absorvem mais de $50 \%$ destes recursos na forma de pagamentos e salários.

Isso implica que uma parcela da mão de obra com alta qualificação é mais responsável para o crescimento das firmas em detrimento de outros insumos. Esta importância relativa está precisamente relacionada com as flutuações de proximidade com a 
fronteira tecnológica. Pesquisas recentes, destacando Vandenbussche, Aghion e Meghir (2006), Wu (2010), Ang, Madsen e Islam (2011), Rocha, Dal-Poz e Silveira (2013) e Basu e Mehra (2014), apontaram que as diferenças tecnológicas condicionam as estratégias das empresas, atribuindo maior importância aos fatores que respondem para uma maior convergência. Nesse sentido, o processo de aproximação com a fronteira gera uma redistribuição entre os fatores, aumentando a importância relativa da pesquisa e dos insumos que melhor complementam estes investimentos. Assim como nas firmas, essas diferenças prevalecem entre as economias, de forma que nos países mais desenvolvidos talpadrão de complementaridade entre insumo-tecnologia apresenta uma sistemática distinta em relação às economias menos desenvolvidas. Tais questionamentos acerca das diferenças entre as economias foram adequadamente abordados nas contribuições de Caselli e Coleman (2006).

Seguindo evidências empíricas similares, Coad $(2008 ; 2011)$ analisou a relação entre os esforços em inovação, medido pelos investimentos em $\mathrm{P} \& \mathrm{D}$ e pelo estoque de patentes, e o índice q-Tobin em uma amostra de firmas nos setores de alta tecnologia ao longo dos anos 1972-1999. Por meio da técnica de RQ, os resultados apontam que as firmas operantes na fronteira apresentam maior valor de mercado e situam-se nos quantis superiores da distribuição, beneficiando-se mais das suas inovações em relação às firmas mais afastadas. Assim, os impactos dos esforços em inovação são menores na cauda inferior da distribuição e consideravelmente maiores nos quantis superiores, sugerindo que as "oscilações de proximidade com a fronteira", a partir dos diferentes quantis, condicionam os resultados dos investimentos no desempenho das firmas.

Em confronto com os resultados aqui elucidados, as evidências de Coad (2008; 2011) indicam que os investimentos em $P \& D$ apresentam um retorno crescente e diferenciado na distribuição condicional de uma métrica de desempenho da firma- $Q$ de Tobin. Assim, as vendas representam outra medida de desempenho que aproxima dos resultados nos autores. Pode-se destacar que diferentes medidas de desempenho vêm apresentando uma possível convergência quanto à aplicação de investimentos específicos, como P\&D.

O Quadro 2 apresenta um resumo comparativo entre as diferentes performances das firmas e sua relação com os investimentos: 


\section{Quadro 2 - Posição nos quantis, distância da fronteira e orientação estratégica}

\begin{tabular}{|c|c|c|c|}
\hline \multicolumn{2}{|r|}{ Firmas mais afastadas da fronteira } & \multicolumn{2}{|r|}{ Firmas na vizinhança da fronteira } \\
\hline \multicolumn{2}{|r|}{ Quantis inferiores } & & Quantis superiores \\
\hline & \multicolumn{3}{|c|}{ Tipos de estratégias empresariais } \\
\hline & Imitação & & Inovação \\
\hline * & Estratégias seguidoras das empresas líderes & * & Estratégias baseadas na eficiência \\
\hline * & $\begin{array}{l}\text { Investimentos destinados à execução de } \\
\text { tecnologias preexistentes }\end{array}$ & * & $\begin{array}{l}\text { Recursos de pesquisa destinados a "deslocar" a } \\
\text { fronteira }\end{array}$ \\
\hline * & Avanços nos métodos básicos de produção & * & $\begin{array}{l}\text { Altos níveis de investimento em P\&D e na geração de } \\
\text { patentes }\end{array}$ \\
\hline * & $\begin{array}{c}\text { Conhecimento baseado na "destruição criativa" - } \\
\text { Mark Schumpeter I }\end{array}$ & * & $\begin{array}{c}\text { Conhecimento baseado na "acumulação criativa" } \\
\text { - Mark Schumpeter II }\end{array}$ \\
\hline
\end{tabular}

Fonte: Elaboração própria.

As evidências apresentadas corroboram com alguns estudos, destacando que as firmas situadas nos quantis inferiores estão mais afastadas da fronteira, tornando o impacto do investimento em P\&D menor do que no caso das firmas situadas na "vizinhança" da fronteira ou nos quantis superiores. Este efeito "proximidade" fica mais evidente na medida em que comparamos o método RQ com QR-VI.

$\mathrm{O}$ baixo retorno vinculado ao investimento em P\&D é explicado parcialmente pelo direcionamento estratégico da firma. As organizações mais afastadas da fronteira e com menor performance podem reduzir seus custos com o avanço tecnológico, assim como podem limitar sua exposição às incertezas de mercado por meio da imitação das firmas líderes, o que desloca o foco estratégico e, consequentemente, o desempenho associado ao seu esforço inovativo (COAD e RAO, 2011). Conforme Coad (2011, p. 1071-1072):

If backward firms do decide to engage in R\&D activity, then we suggest that this R\&D should be directed toward imitation of existing products or practices, rather than the introduction of new products. The closer they become to the frontier, however, the more $R \& D$ they will need to undertake, in order to have sufficient "absorptive capacity" (COHEN and LEVINTHAL, 1990) to keep up with their peers.

Conclusões similares também são destacadas por Falk (2012), em estudo recente envolvendo empresas austríacas em diferentes setores ao longo do período de 1995-2006. Conforme o autor, a elasticidade na razão $\mathrm{P} \& \mathrm{D} /$ vendas com relação ao crescimento das firmas apresenta-se notoriamente superior para as firmas situadas acima da mediana na distribuição do crescimento. Esse efeito diferenciado sugere que a política de inovação tende a apresentar melhores resultados quando o crescimento ocasionado às firmas é suficiente para deslocá-las para cima da mediana na distribuição do crescimento. 
Embora os resultados elucidados nesta pesquisa sugiram que os coeficientes de elasticidade do investimento P\&D nas vendas sejam maiores nas firmas com melhor performance, o impacto do arranjo institucional nas decisões de investimento não é posto em questão. Isto porque políticas de incentivo à inovação pautadas no subsídio da pesquisa, sem mudanças na trajetória estratégica das firmas, podem ser insuficientes no contexto da inovação. Resultados nesse sentido foram apresentados por Aghion e Howitt (2009), destacando os riscos de novas armadilhas (da não convergência) para as economias que não implementaram mudanças endógenas de suas instituições.

\section{CONSIDERAÇÕES FINAIS}

A presente pesquisa buscou analisar a contribuição dos investimentos em $\mathrm{P} \& \mathrm{D}$ no aumento das vendas das firmas, conforme diferentes contextos de performance. Partindo de um modelo de crescimento endógeno da firma, as condições de equilíbrio apontam que o retorno do investimento em P\&D é condicionado pelas flutuações de proximidade com a fronteira tecnológica. Além disso, fatores que afetam diretamente os lucros das firmas repercutem nas decisões de investimento, sendo reforçado pelo "grau de proximidade com a fronteira".

Para capturar a influência da heterogeneidade associada ao desempenho e, possivelmente, aos fatores de proximidade com a fronteira, foi empregada a técnica de regressão quantílica com variáveis instrumentais, considerando uma amostra de 2.000 firmas distribuídas em 40 setores e 46 países.

Ao contrário do estimador padrão RQ de Koenker e Bassett (1978), o pressuposto de regressores exógenos em diversas situações pode não ser válido, especialmente nas séries econômicas. Quando se analisa o impacto dos investimentos em P\&D nas vendas das firmas, fatores como o nível dos lucros e o crescimento podem orientar diversas decisões de investimento, especialmente em pesquisa. Nessas condições, o estimador RQ tradicional passa a apresentar sinais de tendenciosidade e inconsistência, mesmo sob condições de grandes amostras.

Para contornar os prejuízos estatísticos associados à endogeneidade nos investimentos em $\mathrm{P} \& \mathrm{D}$, conforme diferentes pontos da distribuição das vendas, foi empregado o estimador de RQ-VI desenvolvido por Chernozhukov e Hansen (2008). Ao contrário do estimador RQ, a elasticidade dos investimentos em P\&D sob a vendas revelou-se bem superior e com um padrão crescente ao longo da distribuição.

Considerando a amostra total, os resultados revelaram que o método RQ-VI gerou estimativas três vezes maior no primeiro quantil; cinco vezes maior no segundo; seis vezes maior no terceiro; e 12 vezes maior no último quantil, comparando o tradicional 
RQ. Esse viés, ocasionado pela exclusão do controle dos instrumentos sob a variável endógena, revelou-se no mesmo sentido para as firmas atuantes nos setores intensivos de alta tecnologia.

As elasticidades estimadas passaram de 1,02\% no primeiro quantil para $1.55 \%$ no último, contra 0,37\% e 0,17\% (Método RQ). Enquanto as análises preliminares indicariam uma redução na elasticidade ao longo dos quantis, o controle da endogeneidade aponta para um aumento substancial para as firmas de maior performance.

Ao contrário dos resultados apresentados por Montresor e Vezzani (2015), a presente pesquisa demonstra que a endogeneidade dos regressores, caso não seja controlada, pode incorrer em sérios prejuízos na estimação dos parâmetros. Dentre os quais, podem-se destacar a importância relativa do fator mão de obra no crescimento das vendas. Isso conduz a uma avaliação de política equivocada, sugerindo que a expansão do emprego contribui para um crescimento mais rápido que os investimentos em $\mathrm{P} \& \mathrm{D}$.

Este padrão contraria recentes pesquisas, destacando Hall, Lotti e Mairesse (2008, 2013), Coad $(2008,2011)$ e Caselli e Coleman (2006), que apontam um contexto de complementaridade entre insumo-tecnologia. Isto sugere que os investimentos em $\mathrm{P} \& \mathrm{D}$ sustentam um maior crescimento, especialmente, para as firmas de melhor desempenho, ou situadas na faixa superior da distribuição das vendas.

Por fim, esse efeito diferenciado sugere que a política de inovação tende a apresentar melhores resultados quando o crescimento ocasionado às firmas seja suficientemente necessário para deslocarem as empresas acima da mediana na distribuição.

\section{REFERÊNCIAS}

ACEMOGLU, D.; AGHION, P.; ZILIBOTTI, F. Distance to Frontier, Selection and Economic Growth. Journal of the European Economic Association, v. 4, n. 1, p. 37-74, 2006.

AGHION, P. et al. Credit constraints and the cyclicality of R\&D investment: evidence from France. Journal of the European Economic Association, v.10, n. 5, p. 1001-1024, 2012.

AGHION, P.; HOWITT, P. The economics of growth. Cambridge, Massachusetts: The MIT Press, 2009.

AGHION, P.; FESTRÉ, A. Schumpeterian growth theory, Schumpeter, and growth policy design. Journal of Evolutionary Economics, v. 27, n. 1, p. 25-42, 2017.

AGHION, P.; JARAVEL, X. Knowledge spillovers, innovation and growth . The Economic Journal, v. 125 , p. 583 , p. 533-545, 2015.

AGHION, P.; HÉMOUS, D.; KHARROUBI, E. Cyclical fiscal policy, credit constraints, and industry growth. Journal of Monetary Economics, v. 64, p. 41-58, 2014.

AGHION, P.; HOWITT, P.; PRANTL, S. Patent rights, product market reforms, and innovation. Journal of Economic Growth, v. 20, n. 3, p. 223-262, 2015. 
ANDREWS, D.; BUCHINSKY, M. Evaluation of a three-step method for choosing the number of bootstrap repetitions. Journal of Econometrics, v. 103, n. 1-2, p. 345-386, 2001.

ANG, J.; MADSEN, J.; ISLAM, M. The effects of human capital composition on technological convergence. Journal of Macroeconomics, v. 33, p. 465-476, 2011.

BASU, S.; MEHRA, M. Endogenous human capital formation, distance to frontier and growth. Research in Economics, v. 68, n. 2, p. 117-132, 2014.

BAUM, C.; SCHAFFER, M.; STILLMAN, S. Instrumental variables and GMM: Estimation and testing. Stata Journal, v. 3, n. 1, p. 1-31, 2003.

BOGLIACINO, F. Innovation and employment: A firm level analysis with European R\&D Scoreboard data. EconomiA, v. 15, p. 2, p. 141-154, 2014.

BOGLIACINO, F; CORDONA, S. The determinants of R\&D investment: the role of cash flow and capabilities. Working Paper, European Commission's Joint Research Centre (JRC), n. 10, 2010.

BOGLIACINO, F.; PIANTA, M. Profits, R\&D and innovation: a model and a test. Working Paper, European Commission's Joint Research Centre (JRC), n. 05, 2010.

BRESCHI, S.; MALERBA, F.; ORSENIGO, L. Technological Regimes and Schumpeterian Patterns of Innovation. The Economic Journal, v. 110, n. 463, p. 388-410, 2000.

CASELli, F; COLEMAN, W. The World Technology Frontier. American Economic Review, v. 96, n. 3, p. 499-522, 2006.

CASTELLANI, D.et al.Multinationality, R\&D and productivity: Evidence from the top R\&D investors worldwide. International Business Review, v. 26, n. 3, p. 405-416, 2017.

CHERNOZHUKOV, V.; HANSEN, C. An IV model of quantile treatment effects. Econometrica, v. 73, n. 1, p. 245-261, 2005.

CHERNOZHUKOV, V.; HANSEN, C. Inference approaches for instrumental variable quantile regression. Economics Letters, v. 95, n. 2, p. 272-277, 2007.

CHERNOZHUKOV, V.; HANSEN, C. Instrumental quantile regression inference for structural and treatment effect models. Journal of Econometrics, v. 132, p. 491-525, 2006.

CHERNOZHUKOV, V.; HANSEN, C. Instrumental variable quantile regression: a robust inference approach. Journal of Econometrics, v. 142, n. 1, p. 379-398, 2008.

CHERNOZHUKOV, V.; HANSEN, C. The impact of 401(K) participation on the wealth distribution: an instrumental quantile regression analysis. Review of Economics and Statistics, $\mathrm{v}$. 86 , n. 3, p. 735-751, 2004.

CHERNOZHUKOV, V.; HANSEN, C.; JANSSON, M. Finite sample inference for quantile regression models. Journal of Econometrics, v. 152, n. 2, p. 93-103, 2009.

COAD, A. Appropriate business strategy for leaders and laggards. Industrial and Corporate Change, v. 20, n. 4, p. 1049-1079, 2011.

COAD, A. Distance to Frontier and Appropriate Business Strategy. Papers on Economics and Evolution, 2008-07, Max Planck Institute of Economics, Evolutionary Economics Group, 2008.

COAD, A.; RAO, R. Innovation and market value: A quantile regression analysis. Economics Bulletin, v. 15, n. 13, p. 1-10, 2006.

COAD, A.; RAO, R. The firm-level employment effects of innovations in high-tech US manufacturing industries. Journal of Evolutionary Economics, p. 21, n. 2, p. 255-283, 2011. 
COAD, A.; SEGARRA, A.; TERUEL, M. Innovation and firm growth: Does firm age play a role? Research Policy, v. 45, n. 2, p. 387-400, 2016.

COHEN, W. "Fifty years of empirical studies of innovative activity and per-formance". In: HALL, B.; ROSENBERG, N. (Eds.). Handbook of the Economics of Innovation, v. 1, p. 129-213. Oxford, UK: North-Holland, 2010.

COHEN, W.; LEVINTHAL, D. Innovation and learning: two faces of R\&D. Economic Journal, v. 99, n. 397, p. 569-596, 1989.

COMMISSION, E. The 2013 EU Industrial R\&D Investment Scoreboard. 2013. Disponível em: $<$ http://ipts.jrc.ec.europa.eu/>. Acesso em: 13 jul. 2014.

DOSI, G. Technological paradigms and technological trajectories: a suggested interpretation of the determinants and directions of technical change. Research Policy, v. 11, n. 3, p. 147-162, 1982.

DOSI, G.; GAFFARD, J.-L.; NESTA, L. Schumpeterian themes on industrial evolution, structural change and their microfoundations: an introduction. Industrial and Corporate Change, v. 17 , n. 4 , p. $601-609,2008$.

DOSI, G.; MARENGO, L.; PASQUALI, C. How much should society fuel the greed of innovators?: On the relations between appropriability, opportunities and rates of innovation. Research Policy, v. 35, n. 8, p. 1110-1121, 2006.

EFRON, B. Bootstrapping methods: another look at the Jackknife. Annals of Statistics,v. 7, n. 1, p. 1-26, 1979.

FALK, M. Quantile estimates of the impact of R\&D intensity on firm performance. Small Business Economics, v. 39, n. 1, p. 19-37, 2012.

GALVAO, A.; MONTES-ROJAS, G. On the equivalence of instrumental variables estimators for linear models. Economics Letters, v. 134,p. 13-15, 2015.

GRIFFITH, R.; REDDING, S.; VAN REENEN, J. R\&D and absorptive capacity: theory and empirical evidence. Scandinavian Journal of Economics, v. 105, n. 1, p. 99-118, 2003.

GRIFFITH, R.; REDDING, S.; VAN REENEN, J. Mapping the two faces of R\&D: Productivity growth in a panel of OECD industries. Review of Economics and Statistics, v. 86, n. 4, p. 883$895,2004$.

HALL, B. The Financing of Research and Development. Oxford Review of Economic, v. 18, n. 1, p. 35-51, 2002.

HALL, B.; LERNER, J. The financing of R\&D and innovation. NBER Working Paper, n. 15.325, 2009.

HALL, B.; LOTTI, F.; MAIRESSE, J. Employment, innovation, and productivity: evidence from Italian microdata. Industrial and Corporate Change, v. 17, n. 8, p. 813-839, 2008.

HALL, B.; LOTTI, F.; MAIRESSE, J. Evidence on the impact of R\&D and ICT investments on innovation and productivity in Italian firms. Economics of Innovation and New Technology, v. 22, n. 3, p. 300-328, 2013.

HALL, B.; MAIRESSE, J.; MOHNEN, P. "Measuring the returns to R\&D”. In: HALL, B.; ROSENBERG, N. Handbook of the Economics of Innovation, v. 1. Amsterdam: NorthHolland by Elsevier, 2010, p. 1033-1082.

HAYASHI, F. Econometrics. Princeton, NJ: Princeton University Press, 2000. 
HÖLZL, W. Is the R\&D behaviour of fast growing SMEs different? Evidence from CIS III data for 16 countries. Small Business Economics, v. 33, n. 1, p. 59-75, 2009.

KANCS, D.; SILIVERSTOVS, B. R\&D and non-linear productivity growth. Research Policy, v. 45, n. 6, p. 634-646, 2016.

KLEIBERGEN, F.; PAAP, R. Generalized reduced rank tests using the singular value decomposition. Journal of Econometrics, v. 133, n. 1, p. 97-126, 2006.

KOENKER, R. Quantile regression. New York: Cambridge University Press, 2005.

KOENKER, R.; BASSETT, G. Regression quantiles. Econometrica, v. 46, p. 33-50, 1978.

LEE, M.; CHOI, M. The determinants of research and development investment in the pharmaceutical industry: focus on financial structures. Osong Public Health Res Perspect, v. 6, n. 5, p. 302-309, 2015.

MALERBA, F. Sectoral systems of innovations: concepts, issues and analyses of six major sectors in Europe.Cambridge: Cambridge University Press, 2004.

MENDES, S.; SERRASQUEIRO, Z.; NUNES, P. Investment determinants of young and old Portuguese SMEs: A quantile approach. Business Research Quarterly, v. 17, n. 4, p. 279-291, 2014.

MONTRESOR, S.; VEZZANI, A. On the R\&D giants' shoulders: Do FDI help to stand on them? Journal of Industrial and Business Economics, v. 42, n. 1, p. 33-60, 2015.

MONTRESOR, S.; VEZZANI, A. The production function of top R\&D investors: Accounting for size andsector heterogeneity with quantile estimations. Research Policy, v. 44, n. 2, p. 381-393, 2015.

PARENTE, P.; SANTOS SILVA, J. A cautionary note on tests of overidentifying restrictions. Economics Letters, v. 115, n. 2, p. 314-317, 2012.

PINDADO, J.; QUEIROZ, V.; LA TORRE, C. How do firm characteristics influence the relationship between R\&D and firm value? Financial Management, v. 39, n. 2, p. 463-862, 2010.

PIRES, A. R\&D and endogenous asymmetries between firms. Economics Letters, v. 103, n.3, p. 153-156, 2009.

ROCHA, L.; DAL-POZ, M.; SILVEIRA, J. Crescimento econômico e a interação entre capital humano e grau de desenvolvimento tecnológico dos países. Estudos Econômicos, Ministério da Fazenda, Série de Textos para Discussão, Ano 2, n. 12, 2013.

STOCK, J. "Instrumental variables in statistics and econometrics". In:WRIGTH, J. (Ed.). International Encyclopedia of the Social \& Behavioral Sciences. 2. ed. Massachusetts: Elsevier, 2015, p. 205-209.

STOCK, J. H. "Instrumental variables in statistics and economics". In: SMELSER, N. J.; BATES, P. B. (Eds.). International Encyclopedia of the Social and Behavioral Science. v. 11. 2001, p. 7577-82.

TEECE, D. Dynamic capabilities and strategic management. New York: Oxford University Press, 2009.

VANDENBUSSCHE, J.; AGHION, P.; MEGHIR, C. Growth, distance to frontier and composition of human capital. Journal of Economic Growth, v. 11, p. 97-127, 2006.

WU, H. Distance to frontier, intellectual property rights, and economic growth. Economics of Innovation and New Technology, v. 19, n. 2, p. 165-183, 2010. 


\section{APÊNDICE}

Tabela 9 - Distribuição das firmas da amostra por país de origem

\begin{tabular}{|c|c|c|c|}
\hline País de origem & Freq. Abs. & Freq. Rel. (\%) & Freq. Cum. (\%) \\
\hline Australia & 15 & 0,75 & 0,75 \\
\hline Austria & 12 & 0,60 & 1,35 \\
\hline Belgium & 13 & 0,65 & 2,00 \\
\hline Bermuda & 10 & 0,50 & 2,50 \\
\hline Brazil & 8 & 0,40 & 2,90 \\
\hline Canada & 17 & 0,85 & 3,75 \\
\hline Cayman Islands & 49 & 2,45 & 6,20 \\
\hline China & 93 & 4,65 & 10,85 \\
\hline CzechRepublic & 1 & 0,05 & 10,90 \\
\hline Denmark & 25 & 1,25 & 12,15 \\
\hline Finland & 20 & 1,00 & 13,15 \\
\hline France & 75 & 3,75 & 16,90 \\
\hline Germany & 130 & 6,50 & 23,40 \\
\hline Greece & 1 & 0,05 & 23,45 \\
\hline Hong Kong & 5 & 0,25 & 23,70 \\
\hline Hungary & 1 & 0,05 & 23,75 \\
\hline Iceland & 1 & 0,05 & 23,80 \\
\hline India & 22 & 1,10 & 24,90 \\
\hline Ireland & 11 & 0,55 & 25,45 \\
\hline Israel & 15 & 0,75 & 26,20 \\
\hline Italy & 30 & 1,50 & 27,70 \\
\hline Japan & 353 & 17,65 & 45,35 \\
\hline Liechtenstein & 1 & 0,05 & 45,40 \\
\hline Luxembourg & 4 & 0,20 & 45,60 \\
\hline Malaysia & 1 & 0,05 & 45,65 \\
\hline Malta & 1 & 0,05 & 45,70 \\
\hline Mexico & 1 & 0,05 & 45,75 \\
\hline New Zealand & 2 & 0,10 & 45,85 \\
\hline Norway & 11 & 0,55 & 46,40 \\
\hline Portugal & 4 & 0,20 & 46,60 \\
\hline Russia & 4 & 0,20 & 46,80 \\
\hline Saudi Arabia & 1 & 0,05 & 46,85 \\
\hline Singapore & 5 & 0,25 & 47,10 \\
\hline Slovenia & 1 & 0,05 & 47,15 \\
\hline South Africa & 1 & 0,05 & 47,20 \\
\hline South Korea & 56 & 2,80 & 50,00 \\
\hline Spain & 16 & 0,80 & 50,80 \\
\hline Sweden & 40 & 2,00 & 52,80 \\
\hline Switzerland & 54 & 2,70 & 55,50 \\
\hline Taiwan & 82 & 4,10 & 59,60 \\
\hline Thailand & 1 & 0,05 & 59,65 \\
\hline The Netherlands & 35 & 1,75 & 61,40 \\
\hline Turkey & 6 & 0,30 & 61,70 \\
\hline UK & 107 & 5,35 & 67,05 \\
\hline USA & 658 & 32,90 & 99,95 \\
\hline Venezuela & 1 & 0,05 & 100,00 \\
\hline Total & 2000 & 100,00 & \\
\hline
\end{tabular}

Fonte: Elaboração própria. 
Tabela 10 - Distribuição das firmas da amostra por setor

\begin{tabular}{|c|c|c|c|}
\hline Setor (ICB-3D) & Freq. Abs. & Freq. Rel. (\%) & Freq. Cum. (\%) \\
\hline Aerospace \& Defence & 46 & 2,3 & 2,3 \\
\hline Alternative Energy & 8 & 0,4 & 2,7 \\
\hline Automobiles \& Parts & 126 & 6,3 & 9 \\
\hline Banks & 27 & 1,35 & 10,35 \\
\hline Beverages & 10 & 0,5 & 10,85 \\
\hline Chemicals & 117 & 5,85 & 16,7 \\
\hline Construction \& Materials & 52 & 2,6 & 19,3 \\
\hline Electricity & 24 & 1,2 & 20,5 \\
\hline Electronic \& Electrical Equipment & 177 & 8,85 & 29,35 \\
\hline Equity Investment Instruments & 1 & 0,05 & 29,4 \\
\hline Financial Services & 13 & 0,65 & 30,05 \\
\hline Fixed Line Telecommunications & 20 & 1 & 31,05 \\
\hline Food \& Drug Retailers & 3 & 0,15 & 31,2 \\
\hline Food Producers & 51 & 2,55 & 33,75 \\
\hline Forestry \& Paper & 9 & 0,45 & 34,2 \\
\hline Gas, Water \& Multi-utilities & 11 & 0,55 & 34,75 \\
\hline General Industrials & 69 & 3,45 & 38,2 \\
\hline General Retailers & 12 & 0,6 & 38,8 \\
\hline Health Care Equipment \& Services & 83 & 4,15 & 42,95 \\
\hline Household Goods \& Home Construction & 34 & 1,7 & 44,65 \\
\hline Industrial Engineering & 178 & 8,9 & 53,55 \\
\hline Industrial Metals \& Mining & 33 & 1,65 & 55,2 \\
\hline Industrial Transportation & 9 & 0,45 & 55,65 \\
\hline Leisure Goods & 34 & 1,7 & 57,35 \\
\hline Life Insurance & 2 & 0,1 & 57,45 \\
\hline Media & 16 & 0,8 & 58,25 \\
\hline Mining & 10 & 0,5 & 58,75 \\
\hline Mobile Telecommunications & 5 & 0,25 & 59 \\
\hline Nonequity Investment Instruments & 1 & 0,05 & 59,05 \\
\hline Nonlife Insurance & 1 & 0,05 & 59,1 \\
\hline Oil \& Gas Producers & 26 & 1,3 & 60,4 \\
\hline Oil Equipment, Services \& Distribution & 14 & 0,7 & 61,1 \\
\hline Personal Goods & 37 & 1,85 & 62,95 \\
\hline Pharmaceuticals \& Biotechnology & 215 & 10,75 & 73,7 \\
\hline Real Estate Investment \& Services & 2 & 0,1 & 73,8 \\
\hline Software \& Computer Services & 188 & 9,4 & 83,2 \\
\hline Support Services & 19 & 0,95 & 84,15 \\
\hline Technology Hardware \&Equipment & 293 & 14,65 & 98,8 \\
\hline Tobacco & 6 & 0,3 & 99,1 \\
\hline Travel \& Leisure & 18 & 0,9 & 100 \\
\hline Total & 2000 & 100 & \\
\hline
\end{tabular}

Fonte: Elaboração própria. 


\section{Tabela 11 - Distribuição das firmas por tipo de intensidade dos investimentos}

\begin{tabular}{lccc}
\hline R\&D intensity & Freq. Abs. & Freq. Rel. (\%) & Freq. Acum. (\%) \\
\hline High R\&D intensity & 1.473 & 73,65 & 73,65 \\
Medium R\&D intensity & 454 & 22,70 & 96,35 \\
Low R\&D intensity & 73 & 3,65 & 100,00 \\
Total & $\mathbf{2 . 0 0 0}$ & $\mathbf{1 0 0 , 0 0}$ & - \\
\hline
\end{tabular}

Legenda: setores com alta intensidade nos investimentos representam o conjunto de firmas com [R\&DNendas $5 \%$ ]; setores com média intensidade nos investimentos representam o conjunto de firmas com [ $1 \%$ R\&D/Nendas $<5 \%$ ]; setores com baixa intensidade nos investimentos representam o conjunto de firmas com [R\&D/Nendas $<1 \%]$.

Fonte: Elaboração própria. 